\title{
DESIGN OF A NON-CONTACT HOME MONITORING SYSTEM FOR AUDIO DETECTION OF INFANT APNEA
}

\author{
A Thesis \\ presented to \\ the Faculty of California Polytechnic State University, \\ San Luis Obispo
}

\author{
In Partial Fulfillment \\ of the Requirements for the Degree \\ Master of Science in Engineering
}

With a Specialization in Biomedical Engineering

by

Daniel Thomas White

August 2015 
(C) 2015

Daniel Thomas White

ALL RIGHTS RESERVED 
TITLE:

AUTHOR:

DATE SUBMITTED:

COMMITTEE CHAIR:

COMMITTEE MEMBER:

COMMITTEE MEMBER:
Design of a Non-Contact Home Monitoring System for Audio Detection of Infant Apnea

Daniel Thomas White

August 2015

Lily Laiho, Ph.D.

Professor of Biomedical Engineering

Richard Savage, Ph.D. Director of Graduate Education Professor of Biomedical Engineering

David Clague, Ph.D.

Professor of Biomedical Engineering 


\author{
ABSTRACT \\ Design of a Non-Contact Home Monitoring System for \\ Audio Detection of Infant Apnea \\ Daniel Thomas White
}

Infant apnea is a widespread condition in which infants fail to effectively breathe, and can lead to death. Clinical solutions exist for continuous monitoring of respirations in a hospital setting and requiring constant skin contact. This thesis investigates the construction of a proof of concept device that performs inhome monitoring without skin contact and with commonly available off-the-shelf components.

The device constructed used a directional microphone to detect breathing sounds, an omnidirectional microphone to detect ambient noise as a baseline to help isolate the breathing sounds, and LabVIEW software deployed on an inexpensive laptop computer to quantify incidents of apparent lapses in breathing meeting the clinical definition of apnea. Testing results indicate that these components are effective in capturing these events in pre-term infants as well as adults, which provides promising evidence that a low-cost system could be manufactured for home detection to assist in infant monitoring. 


\section{ACKNOWLEDGMENTS}

Several people provided critical assistance through the phases of this project.

First, I would like to thank Dr. Lily Laiho, who initially approached me with the project, assisted in organizing team meetings, and served as my thesis advisor during all phases of this thesis.

Secondly, I thank my project members Lauren West and Scott Garrett, who not only were great sounding boards for all of my ideas contributing to the project, but contributed their own indispensable portions in Lauren's $\mathrm{CO}_{2}$ monitoring module and Scott's design of enclosure, as well as assistance in project updates and presentations.

I would also like to thank Aaron Stein, John Jacobs, and Robert McPhie, the team of engineers at Raytheon who provided consistent feedback and guidance during the design of this project as well as critical funding. In addition, for the connection to Dr. Steven Barkley at Cottage Hospital in Goleta, CA, who I would also like to thank for donating his expertise, time, and access in the Neonatal Intensive Care Unit for our project team to carry out critical tests.

Finally, I am thankful for anyone who offered support and guidance, but most importantly my eternal classmate, colleague, and wife Leah White (Rosenthal), who provided massive support at every step. For the rest of my family, I thank them for pretending hide their disdain during the long road to completion of this thesis. 


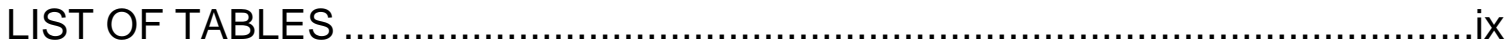

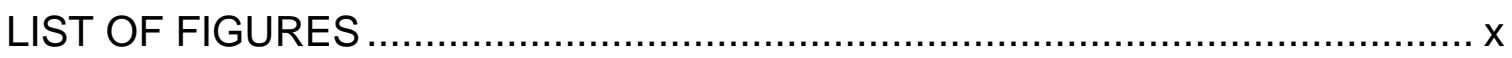

CHAPTER

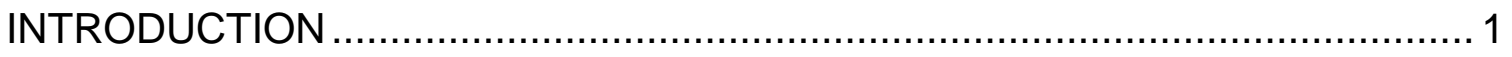

Significance of Infant Apnea ........................................................... 1

Respiratory System and Anatomy Related to Apnea ............................... 1

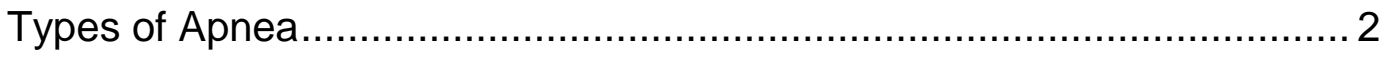

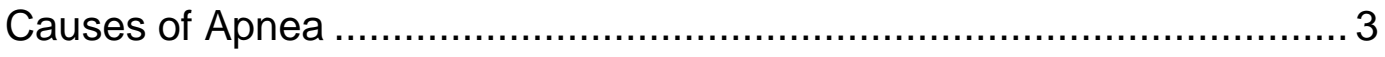

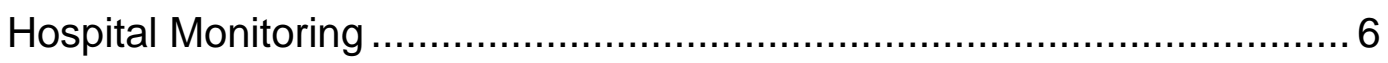

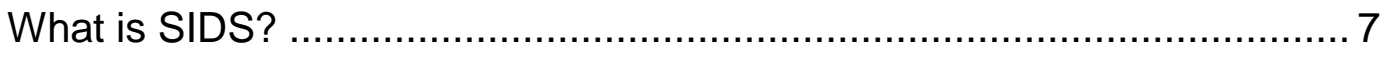

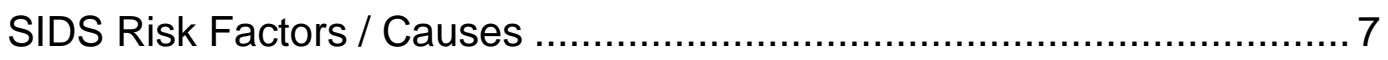

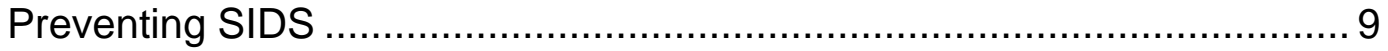

The Relationship Between Apnea, SIDS and ALTE ….......................... 10

What Solutions Exist to Monitor and Protect Infants from Apnea Death? .................................................................... 12

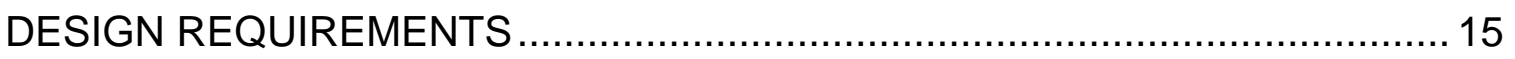

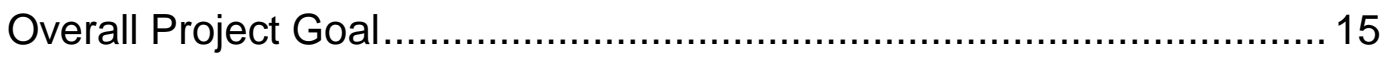

Detection Requirements ....................................................... 15

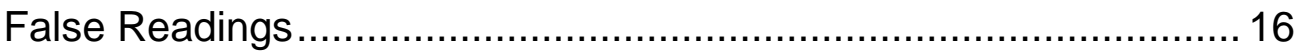

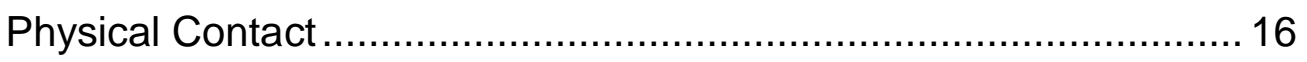


Size and Installation................................................................. 17

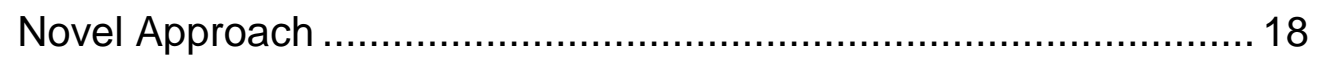

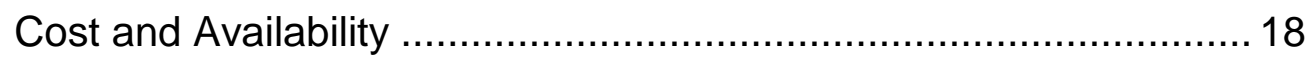

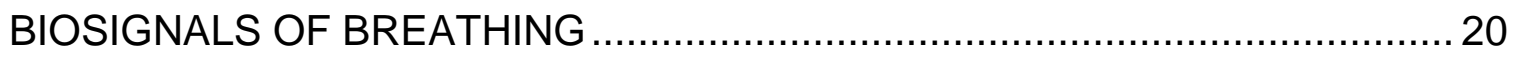

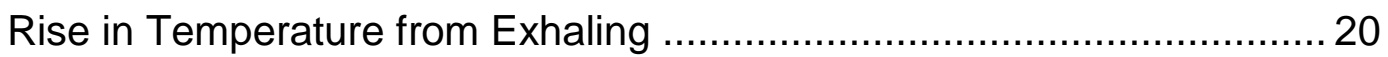

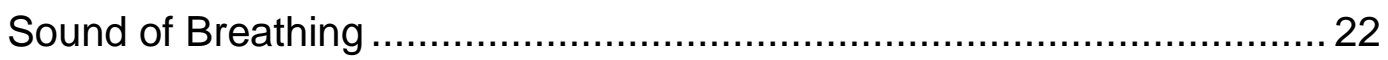

Physical Motion of Chest During Inhaling and Exhaling .........................23

Gas Content in Exhaled Breath ..................................................... 24

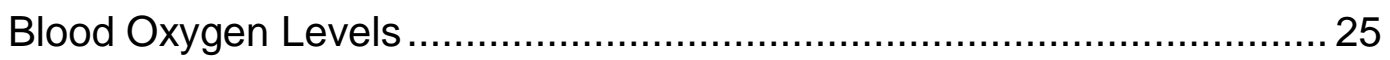

Detection of Bioelectrical Signals Related to Breathing …......................26

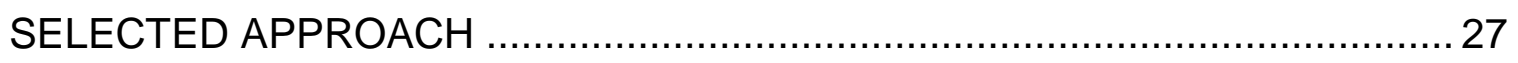

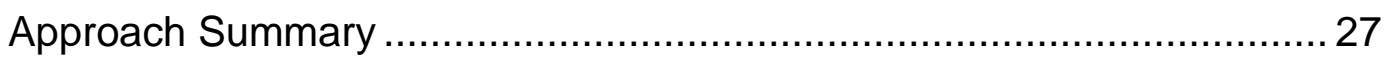

Breath Sounds via Microphone ..................................................... 27

Signal Processing and Integration with $\mathrm{CO}_{2}$ Detection System ........ 28

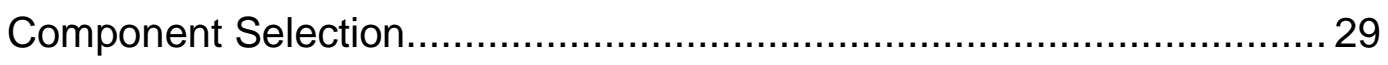

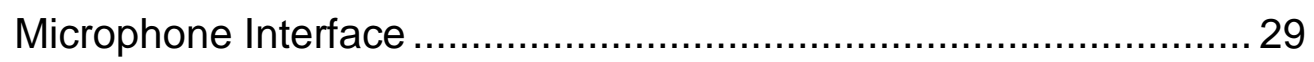

Directional Microphone …......................................................... 30

Omnidirectional Microphone ........................................................ 30

USB Audio Interface / Preamplifier (Audio Input Device) ................... 31

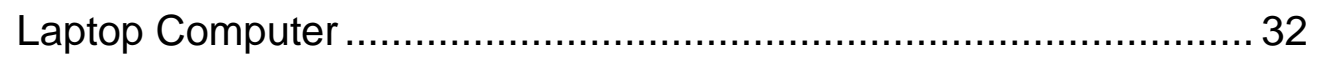

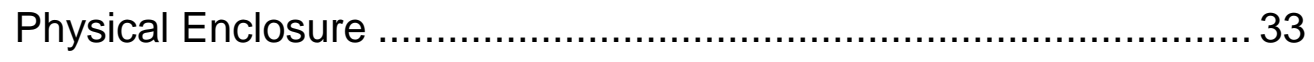

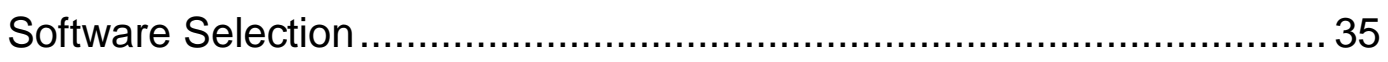


LabVIEW

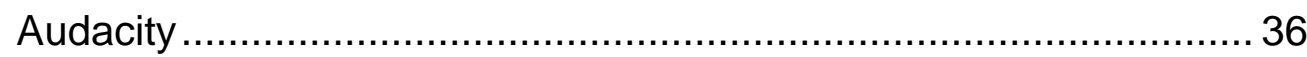

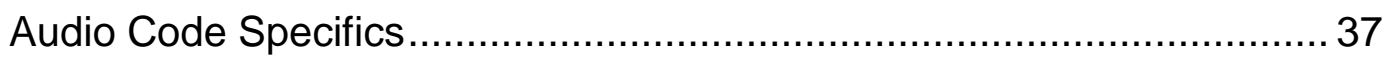

What To Look For in the Audio Signal ........................................... 37

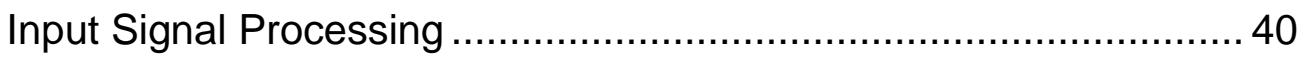

Handling of Processed Signal.......................................................... 44

Front End - Graphs, Charts, and User Interface ............................... 46

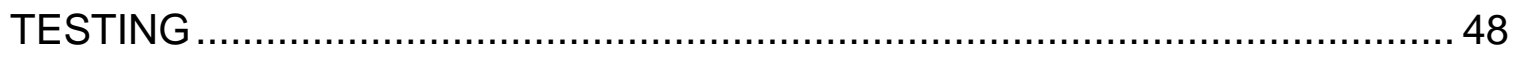

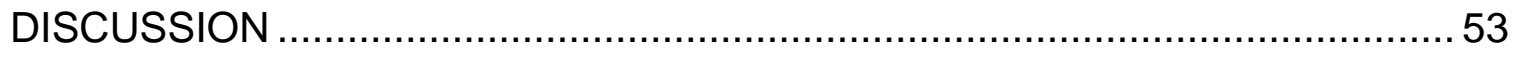

Discussion of Table 2 - Initial Error Testing Results ..............................53

Discussion of Table 3 - Positive / Negative Error Testing Results ...........54

Discussion of Table 4 - Disruptive Ambient Noise Testing Results.......... 55

Discussion of Table 5 - Longevity Testing Results ............................... 55

Discussion of Table 6 - Premature Infant Testing Results .......................56

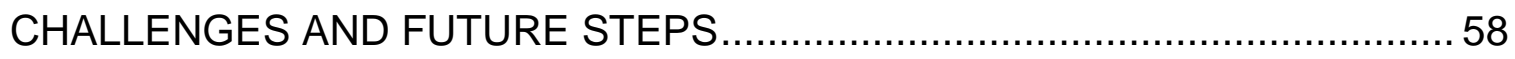

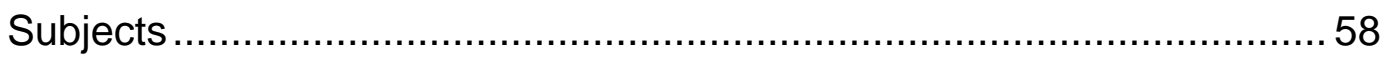

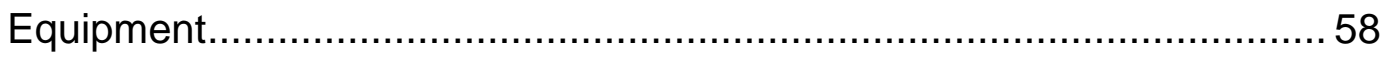

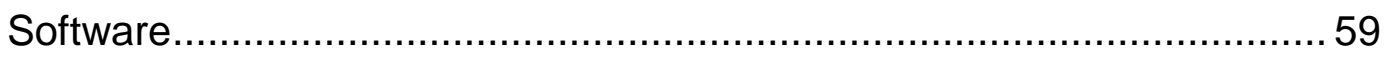

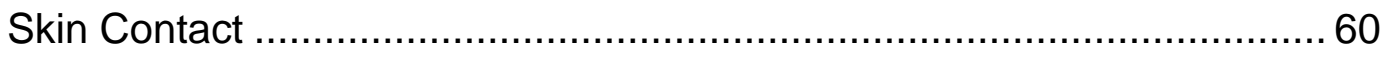

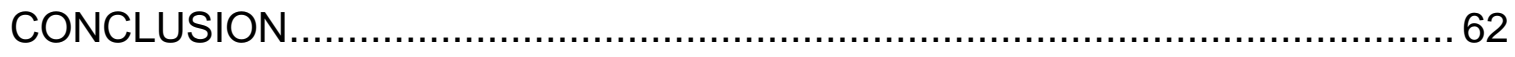

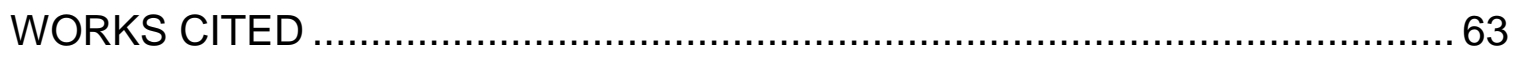

viii 


\section{LIST OF TABLES}

Table

Page

Table 1 - Notation for Intermediate Values in LabVIEW 40

Table 2 - Initial Error Testing Results 48

Table 3 - Positive / Negative Error Testing Results 49

Table 4 - Disruptive Ambient Noise Testing Results......................................50

Table 5 - Longevity Testing Results 51

Table 6 - Premature Infant Testing Results 52 


\section{LIST OF FIGURES}

Figure

Page

Figure 1 - Hardware Components of Audio Detection System

29

Figure 2 - Hardware Enclosure, Underside 33

Figure 3 - Mounted Hardware Enclosure, Front............................................. 34

Figure 4 - Mounted Hardware Enclosure with Microphones, Rear 34

Figure 5 - Spectrograms from Adult and Child Breath Sounds 38

Figure 6 - LabVIEW Code 41

Figure 7 - LabVIEW Code, Continued 45

Figure 8 - LabVIEW User Interface. 46

Figure 9 - LabVIEW Log Interface and Configurable Settings 47 


\section{INTRODUCTION}

\section{Significance of Infant Apnea}

Apnea, a common problem that affects adults and infants, is defined as cessation of respiration for longer than 20 seconds or shorter duration in presence of cyanosis or bradycardia. (Mishra, Agarwal, Jeevasankar, Aggarwal, Deorari, \& Paul, 2008) This paper will focus on infant apnea and present an affordable, non-contact method to detect potential infant apnea in a timely manner to prevent negative consequences. Apnea in infants can occur for many reasons. It can occur somewhat regularly or it may only occur once, but once can be enough to end a child's life.

\section{Respiratory System and Anatomy Related to Apnea}

Since Apnea is the cessation of breathing it is worth taking a little time on the background of breath and the systems that control it. The purpose of the respiratory system is to support getting oxygen to the body and to remove carbon dioxide. This is accomplished through the flow of oxygen into the lungs, through the lung alveoli and then from there into the blood. The lung alveoli also remove carbon dioxide from the blood and transfer it back into the lungs to be expelled.

This process is triggered by the expansion and contraction of the lungs which is controlled by either the movement of the diaphragm or the depression of 
the ribs to decrease the size of the chest cavity. The diaphragm is responsible for the typical quiet breathing that occurs at rest. During the inhale the diaphragm contracts pulling the lungs downward and increasing their volume. Then the diaphragm relaxes which compresses the lungs causing them to exhale.

The nervous system plays a big part in regulating the rate of ventilation to meet the oxygen demands of the body. The nervous signal is transmitted to the inspiratory muscles including the diaphragm to drive inspiration. It delivers a signal that starts out weak and slowly increases before it then ceases which allows the diaphragm to relax causing expiration. This cycle repeats at whatever rate is needed to support current physical activity. (Guyton \& Hall, 2006)

\section{Types of Apnea}

Apnea occurs in infants and adults, although the root causes of these apneas may be different. There are 3 main types of Apnea:

- Central apnea: Central apnea accounts for approximately $40 \%$ of apnea cases. It is defined by a lack of desire to breath, caused by nervous system issues. In these cases there is no obstruction blocking air flow.

- Obstructive apnea: Obstructive apnea accounts for approximately $10 \%$ of apnea cases. It is defined by the existence of a physical 
obstruction in the upper airway.

- Mixed apnea: Mixed apnea accounts for approximately $50 \%$ of apnea cases. It is defined as a respiratory obstruction that occurs directly after the central nervous system causes pauses. It consists of obstructed respiratory efforts usually following central pauses. (Mishra, Agarwal, Jeevasankar, Aggarwal, Deorari, \& Paul, 2008)

\section{Causes of Apnea}

Pre-term infants are at risk of a type of apnea called apnea of prematurity (AOP). This is due to their immaturity of the central nervous system at time of birth. The occurrence is therefore inversely proportional to how premature the infant was at birth. The occurrence rate starts at $60 \%$ for infants who are born in less than 28 weeks, and decreases to $10 \%$ for babies who are carried until 34 weeks before birth. (Mishra, Agarwal, Jeevasankar, Aggarwal, Deorari, \& Paul, 2008) AOP is only diagnosed as the cause for Apnea if other secondary causes can be ruled out, this includes common causes such as sepsis, pneumonia, asphyxia, temperature instability and anemia. (Mishra, Agarwal, Jeevasankar, Aggarwal, Deorari, \& Paul, 2008)

Because of a disconnect in a premature infant's brain development they seem to have a lower response to the buildup of carbon dioxide that typically causes the instinct to breathe. This occurs because the brain of a preterm infant 
has a decreased number of synaptic connections, dendritic arborizations and poor myelin. These all affect the health and effectiveness of the central nervous system. This has been shown in previous studies where auditory responses in infants who have apnea are delayed which points to decreased brainstem conduction time. (Abu-Shaweesh \& Martin, 2008)

This decrease in functionality of the nervous system leads to increased chances of mixed or central apnea. The response to hypoxia that causes the inspiration to breath is caused mostly by the peripheral chemoreceptors located in the carotid body. If these chemoreceptors under function or over function it can lead to apnea, or bradycardia. (Wildhaber \& Moeller, 2007) Bradycardia is often associated with preterm infants with apnea; this often presents itself as an absence of QRS complex for 3 seconds when observed in an EKG. (AbuShaweesh \& Martin, 2008)

Another factor contributing to apnea is that in utero the infant's chemoreceptors are adjusted to a level of oxygen that is normal in a fetal environment. After birth the chemoreceptors change but this can take some time. (Abu-Shaweesh \& Martin, 2008) The necessary chemoreceptor changes are typically complete 2 weeks after birth in both full term and preterm situations. (Abu-Shaweesh \& Martin, 2008) Sometimes in preterm infants this period of time where the central chemo sensitivity is impaired can be longer. It has been seen to make a difference with the response to carbon dioxide compared to full term infants or adults. This leads to the pre-term infants not responding with the typical 
respiratory reaction of expiring the build-up of carbon dioxide and breathing in oxygen. It has been found that this impairment is more common in infants with apnea than infants who have not had apnea.

Increased oxygen levels that lead to hyperoxia (overly high concentration of oxygen) have been shown to induce apnea by silencing the carotid body. After this the body goes into hypoxia (a lack of oxygen). Hypoxia has been shown to lead to a delayed depression in breathing in pre-term infants; this is thought to be related to the nervous system. This can prolong the apnea episode and extend the time to recovery (Abu-Shaweesh \& Martin, 2008)

Other reasons that infants have a higher risk of apnea have to do with the physical characteristics of their respiratory system. When under one year of age the airway diameter is still very small, the muscles surrounding the airway are not very strong, and the orientation of the rib cage and diaphragm are more horizontal. These structural characteristics lead to a reduced air flow and increased flow resistance. This also means the residual capacity of the lungs is lowered. This becomes a larger issue during sleep. During REM cycle sleep the combination of the weak muscles and compliant chest wall can lead to Paradoxical breathing, which is when the diaphragm muscle moves in the opposite direction than it normally would. This can lead to many abnormal and unstable oxygen and gas levels, consumption, and respiratory rate. (Wildhaber \& Moeller, 2007) 
Apnea of Prematurity can also happen when the infant is awake. This is caused when the infant holds its breath for too long, this can happen both while the infant is crying and not crying, and can lead to severe hypoxia. The typical recovery method for this is for the infant to gasp in order to self-resuscitate. Typically this happens when the infant is in some sort of physical or emotional pain or stressful situation. (Thach, 2005)

\section{Hospital Monitoring}

All babies less than 34 weeks gestation should be monitored for at least in the first week of life or until absence of apneic episodes for at least 7 days. Babies more than 34 weeks gestation should be monitored if they are sick. (Mishra, Agarwal, Jeevasankar, Aggarwal, Deorari, \& Paul, 2008)

If it is found that an infant has apnea they are typically checked for bradycardia, cyanosis, and any airway obstructions. Typically tactile stimulation is given and oxygen can be given if needed. There are even drugs that can be used to prevent and treat Apnea of Prematurity. (Mishra, Agarwal, Jeevasankar, Aggarwal, Deorari, \& Paul, 2008) Apnea of Prematurity can lead to something called SIDS (Sudden Infant Death Syndrome) but it is not the only or even main cause of SIDS. In fact only approximately $2 \%-4 \%$ of infants who die of SIDS have had a history of Apnea of Prematurity. Therefore it is important that we look deeper into what causes SIDS as well. (Mishra, Agarwal, Jeevasankar, 
Aggarwal, Deorari, \& Paul, 2008)

\section{What is SIDS?}

SIDS is defined as "the sudden death of an infant under one year of age, which remains unexplained after a thorough case investigation, including performance of a complete autopsy, examination of the death scene, and review of the clinical history". (Moon \& Horne, 2007) SIDS is most common in boys than girls, occurring at a ratio of 60:40. It is least likely in the first month of life, but then increases and is most common around 2-4 months of age. The chances start to decrease after that point. A total of $90 \%$ of SIDS cases occur in the first 6 months of the infant's life. (Moon \& Horne, 2007)

SIDS is the number one cause of death for infants between the ages of 1 month and 12 months old in developed countries. (Moon \& Horne, 2007) Rates of SIDS vary around the world anywhere between .8 and .09 per 1000 live births. The United States has an occurrence rate of 0.57 deaths from SIDS per 1000 live births. (Moon \& Horne, 2007)

\section{SIDS Risk Factors / Causes}

Risk factors for SIDS include mothers smoking during childbirth, sleeping 
arrangement and position, infants being premature or underweight, and possible genetic risk factors. Some recommendations to reduce the risk of SIDS include having infants sleep on their back, avoiding soft bedding, and having a separate but nearby sleeping area for the infant (no bed sharing). (Moon \& Horne, 2007)

The common factors in children dying of SIDS have changed over the past two decades. It seems that a greater proportion of the infants passing away from SIDS were premature and in negative social situations. Bed Sharing, exposure to smoking, and prone sleeping continue to be contributing risk factors even after knowledge of these factors were publicized. In more recent years more of the children dying of SIDS have had identifiable risk factors than what has been seen historically.

Even though bed sharing is a contributing factor it should be noted that there is not a direct correlation between the age of children most commonly sharing a bed with parents, which is 1 month of age, and the most common age for SIDS to occur, which is 2 - 5 months old. It seems that the age that bed sharing seems to have the highest incidence in relation to SIDS is 2 months of age. This could show that the risk factors are only part of the issue, when they combine with the physical changes occurring in the infants at different ages they can increase the chances of SIDS at certain ages.

It has been shown that a few hereditary genetic issues can be tied to SIDS as well. These include: 
- Arrhythmias caused by ion channels issues in the cardiac tissue, like Long QT Syndrome.

- Polymorphisms in serotonin transporter genes.

- Genes that control the development of the autonomic nervous system.

These variants alone cannot cause SIDS but they can be one of the risk factors that combine to lead to it. This is because they would affect a newborn's natural responses to stressors from other risk factors. For example, if an infant was experiencing apnea this could lead to arrhythmias that could be fatal if the infant has Long QT Syndrome. Other abnormalities have been seen in SIDS infants that correlate to some sort of developmental delay that could have been caused by negative events that happened during gestation. These include episodes of hypoxia that can occur more often when women smoke or are exposed to nicotine. This can restrict oxygen to the infant by constricting the uterine vessels. This lack of oxygen can lead to a number of developmental delays. (Leiter \& Böhm, 2007)

\section{Preventing SIDS}

In 1992 the American Academy of Pediatrics launched a campaign called "Back to Sleep" that urged parents to put their babies to sleep on their side or 
back (lateral and supine positions) and not on their stomach (prone position). This campaign was very successful and increased the number of infants put to sleep on their back and side from 28\% in 1992 to approximately $85 \%$ by 2007 . The rate of SIDS decreased from 1.2 deaths per 1000 live births in 1992 to .51 per 1000 live births in a study performed in 2004. But even though the sleeping position seems to be tied to SIDS prevention there is no agreed upon biological reason that prone sleeping should lead to infant death. (Leiter \& Böhm, 2007)

Even though SIDS cannot be treated there are a few interventions that can be done if an infant is found right away. Interventions can include:

- Gentle Stimulation

- Blowing air in face

- Vigorous stimulation

- Mouth-to-mouth breathing

- Cardiopulmonary resuscitation by medically trained person (Hall \& Zalman, 2005)

This is why it is so critical for there to be infant monitoring that can alert others to come to the infant's aid as soon as possible to try and prevent death.

\section{The Relationship Between Apnea, SIDS and ALTE}

For a long time it was thought that Apnea would lead to SIDS but results 10 
of recent studies have shown that it can't be used as a predictor. It has been shown that Apnea monitors can be useful in situations like Apnea, and Apparent Life Threatening Events even though they might not be useful in reducing the actual occurrence of SIDS. (Moon \& Horne, 2007)

Apparent Life Threatening Event (ALTE) is defined by the 1986 National Institutes of Health Consensus Panel on Infantile Apnea and Home Monitoring as a "sudden event, frightening to the observer, in which the infant exhibits a combination of symptoms, including apnea, change in color (pallor, redness, cyanosis, plethora), change in muscle tone (floppiness, rigidity), choking, gagging, or coughing." The occurrence of ALTEs is difficult to accurately determine because the data is only taken from hospital admittance information but a portion of children who experience ALTE are never brought in to be evaluated. The reported numbers show an occurrence rate of $0.05-6 \%$ with most occurring in children under one years of age. (Hall \& Zalman, 2005)

Studies have shown that roughly 4-10 percent of children who die of SIDS have experienced reported ALTEs. It is thought that the actual percentage is higher since most ALTEs are not reported. This thought is backed up by a study that focused on the children of nurses which showed that 27 percent of on children who have died of SIDS had experienced ALTEs. The chances of SIDS occurring in children who have experienced an ALTE increase if the ALTE was related to a cardiac arrhythmia, seizures, or central hypoventilation. It is recommended for children who have experienced a sever ALTE to use some sort 
of home monitoring. There is no known way to prevent ALTEs at the moment. There has been no decrease in the rate of ALTEs using the "Back to Sleep" campaign that has helped with SIDS rates. (Hall \& Zalman, 2005)

\section{What Solutions Exist to Monitor and Protect Infants from Apnea Death?}

Even though there are apnea monitors on the market for both hospital and home use, they each have their pros and cons. There are two main types of monitoring groups, monitors that require contact, and monitors that do not require contact.

The most popular contact monitors include:

- Pulse oximeters - monitors changes in heart rate but have no way to detect chest wall movement.

- Movement sensors- track chest wall movement to ensure that the breathing has not stopped but they can often confuse other body movements as breathing and can miss diagnosis of obstructive apnea.

- Thoracic Impedance Monitors - track the change in thoracic impedance that occurs during breathing, but they can also miss obstructive apnea.

- Respiratory Inductive Plethysmography - tracks both abdominal 
and thoracic movement during breathing. Examples include Abdominal thoracic bands or the Graseby capsule (Mishra, Agarwal, Jeevasankar, Aggarwal, Deorari, \& Paul, 2008)

Most non-contact monitoring systems have been experimental. But they have all shown issues including size, affordability, appropriate sensing and diagnosis, etc. Some types of monitoring that have been explored are:

- Doppler Radar Technology using existing baby monitors to measure cardio and pulmonary rhythms - A low cost system was created using existing baby monitors to output a radio signal and measure the Doppler output signal that was created as this radio signal reflected off of the subject in order to measure chest motion. They found that the radio signal reflected from the body is phase modulated by chest motion and it can therefore be demodulated by a sensor node and then digitized for analysis. They compared this data with data collected by a chest belt and finger sensor that monitored heart rate and chest movement. They found that this data correlated and could be used to determine the chest wall movement. This study did not appear to test if the monitoring system could detect infant breath vs. adult breath or if it could tell when the subject stopped breathing. There was also no alarm 
system built in to this monitoring system. (Hafner, Mostafanezhad, Lubecke, Boric-Lubecke, \& Host-Madsen, 2007)

- Pressure sensor maps built in to mattress to sense sleep events such as apnea - A sensor matt composed of optical pressure sensors is put under a mattress to collect data while the subject sleeps. Pressure data is created by the movement of the chest during breathing. This data is then translated into visual data which produces an interframe similarity matrix that is the main input for this method. Once this is established the pressure caused by breathing translates to a rectangular pattern on the matrix. The size of this rectangle then correlates to the strength of the breath. This allows this approach to sense if the subject is breathing normally or goes in to shallow breathing. This novel approach showed promising results but because of the One major source of error is the low signal to noise ratio for the bed sensor. (Malakuti \& Albu, 2010)

- Diagnosing Apnea through environmental sensors using imaging technology. (Nishada, Mori, Sato, \& Hirai, 2009) 


\section{DESIGN REQUIREMENTS}

\section{Overall Project Goal}

The intent of this project is to build upon previous examples of apnea monitors by creating a proof of concept device that uses novel technologies and is not in physical contact with the subject. In addition, the project will create a system that is affordable using off the shelf components. By doing so, it is the goal of this project to demonstrate the potential for future products to effectively monitor subjects and reduce the health risks associated wtih apnea.

Following this paragraph are more detailed design requirements which outline the specific needs of the system.

\section{Detection Requirements}

The device must be capable of recognizing a lapse in breathing of 20 seconds from an adult or infant. The purpose of this requirement is to ensure that the device's detection criteria matches the clinical definition of apnea. 


\section{False Readings}

The number of false negatives must be as close to zero as possible. This is to say that there should be no events in which the subject is not breathing and the device indicates the subject is breathing. The purpose of this requirement is to ensure that the device successfully prevents the health risks associated with apnea, e.g. death.

Conversely, the number of false positives must be limited as well. This means that the device should not incorrectly recognize a breathing subject as experiencing apnea. Because a false positive does not pose a health risk, this requirement is considered to be a secondary priority to the false positive. As wotj any monitoring device, the purpose of this requirement is to ensure that both the device is functional and build user confidence in a positive readings, which will support a prompt response from caregivers while preventing apathy from "crying wolf".

\section{Physical Contact}

The device must not make physical contact with the subject, and therefore perform all monitoring remotely. The purposes of this requirement are to eliminate concerns of biocompatibility and irritation that may arise from constant 
skin contact, especially on delicate or sensitive skin that would be found on an infant. Additionally, the lack of contact minimizes the chances that a subject may handle the device and inadvertently alter the functionality or safety. The collateral benefit of this requirement is that it increases the number of potential applications for this project in other venues outside of the scope of apnea monitoring, which may be explored in future projects.

Alarm

The device must be capable of emitting an alarm upon detection of an apnea event. For the purposes of this design requirement, it is assumed that this alarm will need to be loud enough to wake a sleeping caretaker over nighttime ambient noise found in an average household. This requirement ensures that the alarm functions to notify caretakers in order to give them the option of timely intervention. In order to satisfy this requirement, the device must be able to emit an alarm of 80 decibels or above, or be modular in such a way that it can trigger an unintelligent device to do the same (for instance, speakers).

\section{Size and Installation}

The device must be sized such that it can comfortably fit inside of an average bedroom. It must also be functional using only facilities found in an 
average bedroom, which includes standard 110V US power outlets. The purpose of this requirement is to ensure that the device could be easily installed in a home, making it more attainable for the general public.

Novel Approach

The approach must be novel, using new concepts of detection and not simply repeat an existing design, commercial or otherwise. The purpose of this requirement is to ensure that the project serves as a proof of concept that opens new possibilities for detection.

\section{Cost and Availability}

All components must be available off the shelf and from a common retailer such as Amazon.com. In addition, they must be chosen to minimize cost as much as possible. The total cost of the system should not exceed $\$ 500$. The total development budget is $\$ 3000$, but the finished product should cost considerably less than that, and $\$ 500$ was selected as a target. The purpose of these requirements is to show that the device could be afforded by many households and is simple enough that it does not require specialized equipment. These requirements also force the design to be somewhat of a worst case scenario; improvements could be made in the cost via mass production and improvements 
in the detection accuracy could be made from the use of more precise equipment. 


\section{BIOSIGNALS OF BREATHING}

In order to approach the problem of detecting breathing, it is important to first examine the possible biosignals, or biological changes that occur during breathing, as well as the current solutions capable of detecting these changes.

\section{Rise in Temperature from Exhaling}

Thermal imaging (FLIR) creates a visual representation of thermal energy across a fixed zone in the line of sight of the device. Areas in the resulting image at higher temperature, such as the air immediately exiting the mouth or nose of the subject, would show up with a visibly distinct color from the surrounding areas on the face or bed. Using image analysis software, this color change could be quantified to trigger a "breath" reading.

Alternatively, infrared thermometers also measure thermal energy, but instead of creating a 2-d map of the thermal energy over an area, they provide a single number reading of the energy in a single, smaller point. If aimed at a portion of the face that changes temperature significantly when exhaling, the output value from an infrared thermometer could be used as a trigger for a breath.

Most of the advantages are common to both thermal imagers and infrared thermometers. Both have enough resolution to detect the temperature 
differences that happen as a result of exhaling. They are capable of meeting the design requirements for unit size and detection distance from the subject.

Thermal imaging has the added advantage of being able to track temperature changes in specific zones across a larger area. With sufficient software, the thermal imaging system could be used without requiring any repositioning or aiming of the camera. Infrared thermometers also meet the requirements for cost. Thermal imaging or infrared thermometers would also work for both obstructive apnea and central apnea because they measure actual respiration, not movement.

Unfortunately, thermal imaging technology is considerably above the price requirements. Thermal imaging also requires additional software in order to process the signal into objective data usable by an integrated detection system, which may require more time and skill than the project allows. Since infrared thermometers only provide a single temperature reading, they need to be pointed solely at the zone on the patient's face that is changing temperature when breathing. This means that if the subject moves, the thermometer would also have to move or else lose the signal and produce a false positive. In addition, the fact that the thermal signature of a breath is held within the air means that air currents from common household fans could completely disrupt the reading. 


\section{Sound of Breathing}

The act of breathing creates sound waves that are audible and detectable by consumer-grade microphones. The volume of breath sounds can vary wildly based on the individual, but are generally around $10 \mathrm{~dB}$. Microphones are available with a variety of detection patterns that can attenuate noise from certain areas, depending on the design. Directional (also known as shotgun) microphones attenuate noise from off-axis sources, meaning the microphone will mainly pick up sound from the direction it is pointed. Omnidirectional microphones do not attenuate sound from any direction. Software could be used to analyze the volume levels from a specific source and conclude that a change in volume came from breathing sounds, then trigger a detection event. Microphones are available at a wide range of prices, many of which are well within the budget guidelines. Sound analysis is not CPU-intensive and can feasibly be performed real-time without advanced software knowledge. The sound spectrum captured by microphones is wide and may contain information leading to accurate detection of breathing, isolating ambient noise. Microphones can be positioned easily in a many different locations and can be effective at detecting breath sounds in the desired range. They also interface easily with signal acquisition software using consumer-grade equipment. 
The majority of the sound spectrum captured by microphones is likely to be noise that will not vary based on the sound of breathing, and therefore will have to be ignored from the signal analysis. In addition, the sound of breathing may be very faint or hidden behind signal noise, which would require creative signal processing or additional hardware to correctly characterize and quantify. It is possible that consumer-grade microphones and sound acquisition hardware may not be sensitive enough to overcome these limitations for a reliable reading. In addition, the sounds generated by a patient struggling during obstructive apnea may be too similar to the sounds from breathing and create a false negative.

\section{Physical Motion of Chest During Inhaling and Exhaling}

Consumer-grade motion detectors used for lighting automation typically work by using passive infrared (PIR) sensors, which work similarly to the previously discussed infrared sensors, but provide a much simpler on/off signal based on the sudden change in heat from movement. They could theoretically be used to detect the motion of an infant's chest, which would signify breathing.

Some advantages of motion detectors that they are inexpensive and could easily be integrated into a software system. They output a simple signal that would not require any software to interpret. 
However, these motion detectors are not designed to be able to detect the small amount of change shown from a subject's chest motion, especially if the subject was an infant. In addition, chest motion may trigger a false negative if the subject is experiencing obstructive apnea and is struggling. In fact, any motion from the subject may trigger this detector.

\section{Gas Content in Exhaled Breath}

The gas content of exhaled air is measurably different from atmospheric air, with the largest composition change in the partial pressures of oxygen and carbon dioxide. Carbon dioxide content can be detected with relatively inexpensive hardware that provides a simple number output, which can be calibrated to indicate the presence of exhaled air.

$\mathrm{CO}_{2}$ detection is relatively immune to false negatives because there are no other sources of $\mathrm{CO}_{2}$ in normal sleeping quarters. It also would be effective in detecting both obstructive and central apnea.

Unless the $\mathrm{CO}_{2}$ detector is located very close to the subject's face, the air composition at the detection location may take a considerable amount of time to change enough to confidently indicate breathing or a lack of breathing. This delay would need to be taken account in determining when to trigger an alarm. For instance, if it takes the $\mathrm{CO}_{2}$ sensor 10 seconds from the cessation of breathing to 
get a confident reading indicating decreased $\mathrm{CO}_{2}$ levels, then the length of time in that zone before sounding the alarm must be 10 seconds shorter than the clinical definition of apnea in order to account for that potential delay.

\section{Blood Oxygen Levels}

Pulse oximetry is a method of detecting blood oxygen levels by comparing the wavelength of absorbed light after being shone on the subject's skin.

Blood oxygen saturation is part of some clinical definitions of apnea and this information would provide concrete evidence of a decrease in blood oxygen. None of the other investigated detection technologies are capable of measuring this information.

Although considered noninvasive, measuring blood oxygen with pulse oximetry requires skin contact, and therefore does not meet the design requirements. A method for measuring this information at any reasonable distance currently has not been developed. 


\section{Detection of Bioelectrical Signals Related to Breathing}

When the muscles responsible for breathing contract, they create a electromyographic (EMG) signal that could be measured to determine when the subject puts physical effort into breathing.

This signal is unambiguous and would be subject to a relatively small amount of noise that might make the signal uninterpretable.

No off the shelf methods for measuring EMG signals without direct skin contact currently exist. In addition, this would only detect central apnea, as a subject experiencing obstructive apnea may still contract breathing muscles while failing to breathe. 


\section{SELECTED APPROACH}

\section{Approach Summary}

\section{Breath Sounds via Microphone}

The most functional and adaptable biosignal detection system within the design requirements is microphone-based audio recording. The act of breathing produces a sound which can be very faint, especially in the case of a small newborn, but detection is still within the realm of possibility with consumer-level equipment.

The detection of breath sounds can be performed without any contact and from a distance that meets the design requirements. It is also inexpensive enough and accurate enough to meet the detection and cost requirements.

Early iterations of this design attempted to use a single directional (supercardioid) microphone in an attempt to detect only the sound coming from the subject using the microphone's built-in property of relatively attenuating noise from other parts of the room, but it was discovered that loud enough noises such as a passing car or creaking chair could not be reliably excluded by the mechanisms in a directional microphone alone. This design is discussed further in subsequent sections. 
The final design for sound detection was to use a directional microphone to obtain data that heavily favors the sound from the subject and pair it with an omnidirectional microphone which detects all sound in the room equally. By subtracting elements in the omnidirectional sound input from the directional sound input, a resulting differential signal is generated that successfully isolates the intended signal from ambient noises.

Signal Processing and Integration with $\mathrm{CO}_{2}$ Detection System

The differential sound input will be generated using LabVIEW software that can take the two sound inputs, perform filtering and subtraction operations, and objectively determine if a breath sound has been detected. In LabVIEW, the code can be created such that it is highly modular, allowing for new detection systems to be integrated alongside the sound detection. With the addition of other biosignal detection systems, the LabVIEW code can have higher confidence in the validity of the detection.

To take advantage of this opportunity, this system also integrates a $\mathrm{CO}_{2}$ discussion system that was created by Lauren West, using a wholly different method of detecting the presence of breaths to add confidence to the sound software. For more information on this detection system, see "A Standoff Approach to Monitoring Infant Apnea" (West, 2010). 
LabVIEW software is capable of running on low-end computer hardware, which enables the use of small and inexpensive systems. It is also capable of accepting many different signal inputs, including those from inexpensive USB adapters. Furthermore, LabVIEW has many options for user interface design, including simple options for visual and audio feedback that will allow this design to have an effective alarm.

\section{Component Selection}

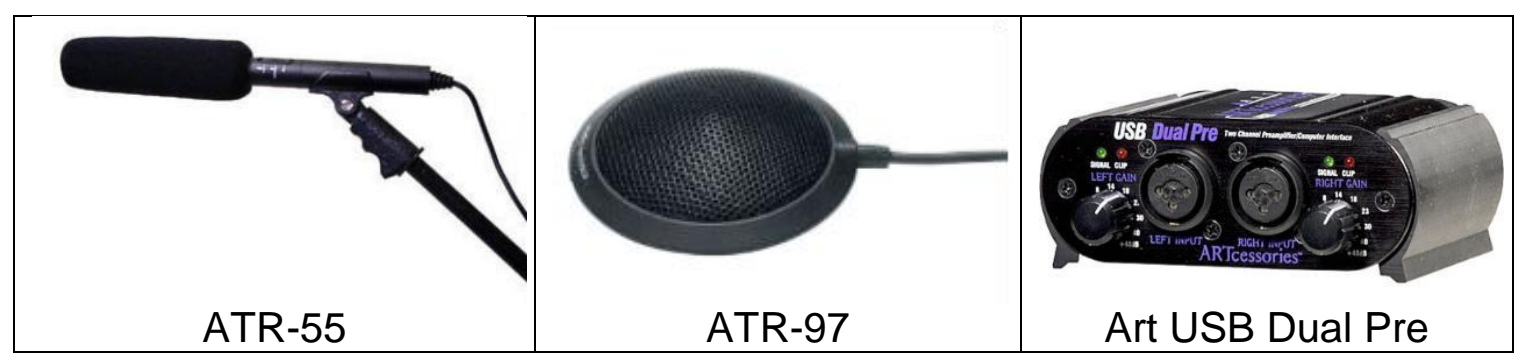

Figure 1 - Hardware Components of Audio Detection System

\section{Microphone Interface}

The two microphones will connect to the Audio Input Device through either XLR or TRS inputs. TRS inputs can be either $1 / 4$ " or $1 / 8$ ". All of these options deliver similar quality for the purposes of this design. XLR inputs are larger and generally more expensive, but would be acceptable. Conversion between $1 / 4$ " and 1/8" TRS connectors is simple and inexpensive. 


\section{Directional Microphone}

The directional microphone must have a sensitivity pattern such that when placed between 1 and 5 feet away from the subject the microphone will attenuate sound outside of where the patient might be able to move during sleep. For the purposes of this project, it is assumed to be the size of a normal infant crib, and ideally the microphone's sensitivity pattern would be such that sounds within the entire area of the crib would be amplified and sounds outside of the area of the crib would be attenuated while the microphone is mounted on the edge of the crib. For this project, the Audio Technica ATR-55 was selected, which has sensitivity characteristics in "tele" mode of $-45 \mathrm{dBm}, 1 \mathrm{kHz}$ at $1 \mathrm{~Pa}$.

\section{Omnidirectional Microphone}

The omnidirectional microphone must have a sensitivity pattern such that it does not attenuate noise from any angle. This microphone is intended to pick up ambient noises and noises that are from outside of the immediate area of the subject. For this project, the Audio Technica ATR-97 was selected, which has sensitivity characteristics of $-46 \mathrm{~dB}, 1 \mathrm{kHz}$ at $1 \mathrm{~Pa}$. 
USB Audio Interface / Preamplifier (Audio Input Device)

The Audio Input Device must be able to accept at least 2 standard microphone inputs of $1 / 8$ " TRS (mini plug), $1 / 4$ " TRS, or XLR inputs. The higher average cost of microphones with XLR connectors makes a device with only XLR inputs not ideal. Either $1 / 4$ " or $1 / 8$ " TRS inputs are equally effective because converters between these two sizes are effective, inexpensive and compact, whereas converting to XLR is generally bulky and more expensive. For these reasons, we focused on devices accepting TRS inputs.

The fundamental feature of the Audio Input Device is an Analog to Digital Converter (ADC) that takes the microphone inputs, processes them, and converts them to a digital signal. The only acceptable connection for transferring this signal to the computer system is USB. A USB connection will ensure compatibility with any modern computer system and is most readily accessed by software in any operating system. By outputting a digital signal to the computer, this limits the number of opportunities to introduce noise into the system from repeated analog operations, which might obscure the important biosignals.

The device will ideally be powered by the USB connection to the computer system alone. Not only does this minimize the number of wires that could potentially become tangled on the crib, it makes it simpler to deal with potential battery backup scenarios. 
The device must have a combination of gain and signal to noise ratio high enough that when combined with the microphone, breath sounds can be detected reliably. Whether or not the device meets this requirement can only be determined from testing.

The Art USB Dual Pre was selected. It can be powered by the USB bus and accepts 2 channels of XLR or $1 / 4$ " TRS audio inputs. These inputs are registered as a right and left channel by the PC it is connected to. The preamplification provided by this unit is low-noise and provides up to $48 \mathrm{db}$ of gain, which is sufficient for amplifying the very quiet breath sounds enough to be clearly heard and analyzed by software. This device also does not require any special drivers or software to be used as an audio device on Windows XP.

\section{Laptop Computer}

A Windows PC is necessary to accept the USB inputs from the Audio Device and run the LabVIEW software, as well as accept connections from any other detection mechanisms that may connect to the software system. All current Windows PCs have the required USB ports and LabVIEW has very low system requirements, so for this project a Dell Mini laptop (also referred to as a "Netbook") was selected. The small size and weight of this laptop allows it to be mounted almost as easily as the rest of the components, and gives the option to integrate it into a complete enclosure. A larger laptop or a desktop computer 
would be much more difficult to mount. In addition, using a laptop with battery power allows the system to maintain functionality during home power loss of 3 hours or less, which is not a design requirement but is a distinct advantage over other home monitoring systems.

Physical Enclosure

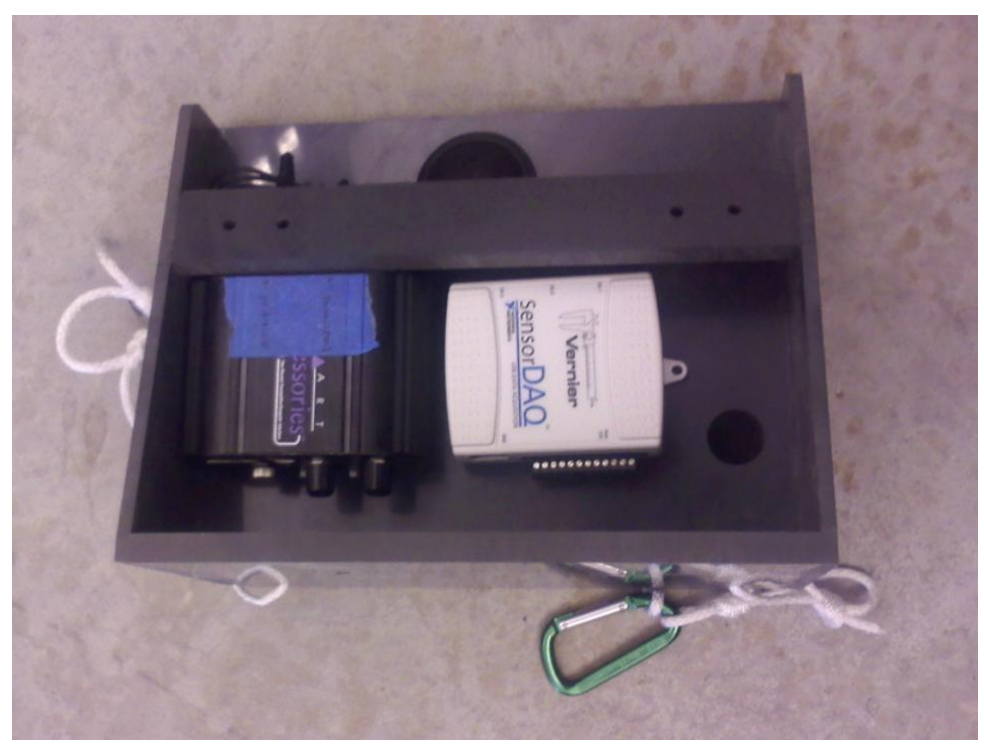

Figure 2 - Hardware Enclosure, Underside 


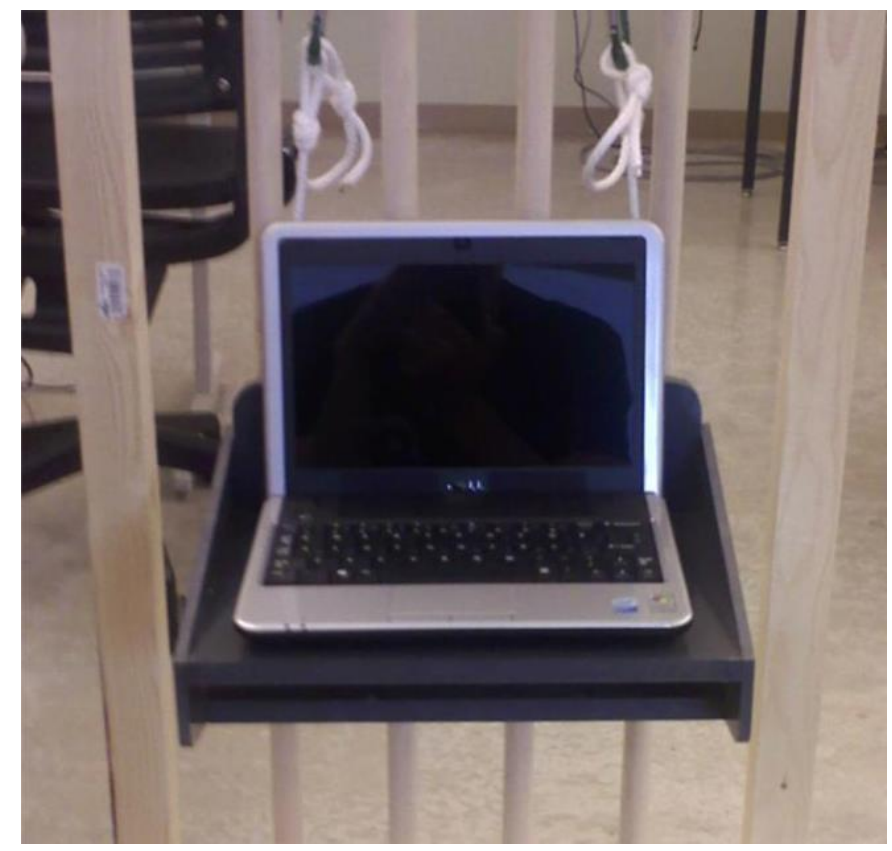

Figure 3 - Mounted Hardware Enclosure, Front

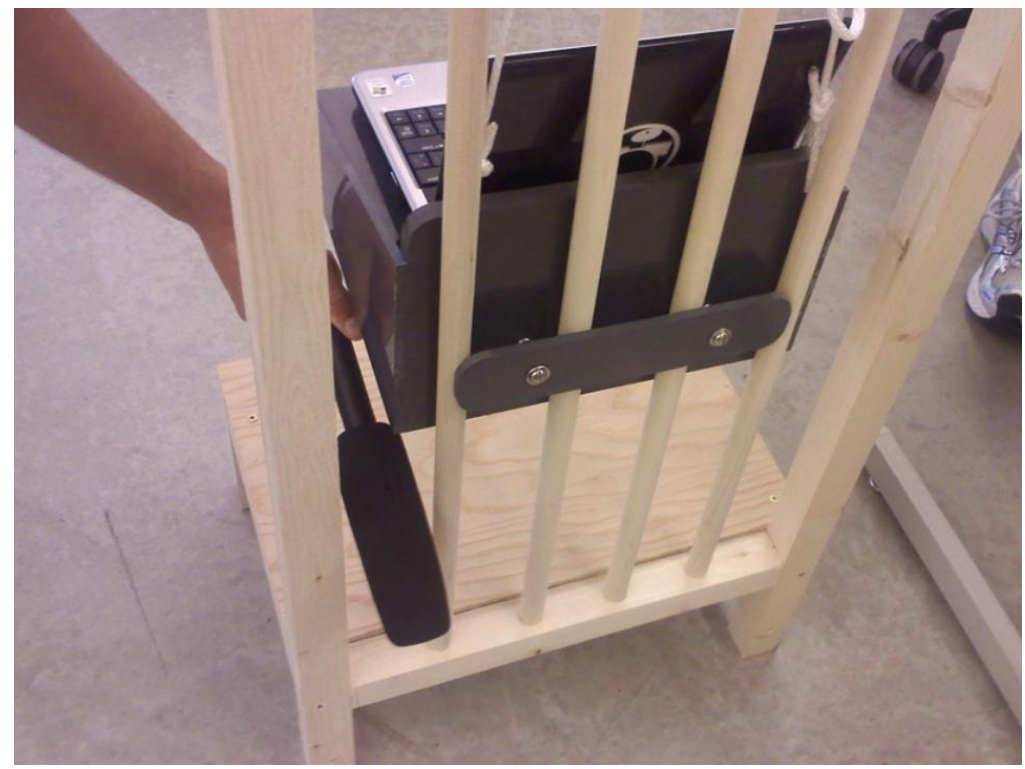

Figure 4 - Mounted Hardware Enclosure with Microphones, Rear 
The enclosure that houses the microphones, laptop, and $\mathrm{CO}_{2}$ detection system was designed by Scott Garrett and is designed to attach to a bedside table or crib. Figure 2, Figure 3, and Figure 4 demonstrate how all of the components of the detection system are mounted and positioned. The directional microphone is ideally positioned as close as possible to the subject, and the omnidirectional microphone is positioned nearby.

\section{Software Selection}

\section{LabVIEW}

LabVIEW software is capable of receiving virtually any input type with the right adapter, which makes it ideal for analyzing the input from diverse sources. In this case, it is processing inputs from a USB audio device and a Vernier DAC, but it could be adapted to use additional signals as well. The USB audio input does not require any special adapters to be usable by LabVIEW, and is suitable for the measurement equipment in the scope of this project.

LabVIEW has built-in functions to perform virtually any mathematical operation on the input signal. It includes analysis tools for separating the audio 
tracks, performing Fourier transforms, as well as other characterizations of the input signal. It can also be augmented with plugins if a necessary function is not found in the standard program. This will be sufficient for processing the audio inputs.

LabVIEW is capable of actuating outputs based on a programmed set of conditions. This includes the creation of log files and the activation of an alarm system. For the scope of this project, we will not be creating an alarm system, but it could be created with little difficulty using the LabVIEW software.

LabVIEW can be used to encapsulate multiple signal analysis systems such that they can be easily integrated with one another. For instance, the sound detection system can be used standalone, or can be used to generate a simple Boolean output that is then weighed against Boolean outputs from other detection systems before determining whether or not to sound the alarm or write a log file.

\section{Audacity}

Audacity is a free audio editing software program that is capable of displaying an audio spectrogram and selectively filtering out ranges of sound. This program is not used for real-time analysis of the input signals, but can be 
used to determine the optimal frequency ranges for each microphone, which can then be used to configure filters in LabVIEW.

\section{Audio Code Specifics}

\section{What To Look For in the Audio Signal}

Before the audio signal can be used by the LabVIEW software to determine the presence of breath sounds, it must first be treated to increase the accuracy of detection. Each microphone will have a unique frequency response to a given sound, and each subject with have a unique frequency of sound produced by their breaths. The sound of breathing will even vary from breath to breath or slowly over the course of sleeping. On top of this, ambient noises in the surrounding area will also be detected by the microphones, which generate amplitudes in frequencies that are irrelevant to the measurement of breath sounds. All of these factors can combine to make the breath sounds more difficult and less accurate to detect, and as a result, the signal must be conditioned.

In order to determine the parameters of the signal conditioning, we first examined a raw spectrogram output of recorded breath sounds. A spectrogram is a method of visualizing three dimensions of a sound signal (amplitude, 
frequency, and time) on a 2-dimensional screen or printout in which the x-axis is time, the $y$-axis is frequency, and the color at a given point indicates the amplitude. Audacity software was used to record sound and generate the spectrograms using the selected microphones and preamplifier.

From the data in Figure 5, we can see that it contains regions that visibly change from the breath sounds and regions that remain unchanged by breath sounds or change from sounds other than the breath sounds.
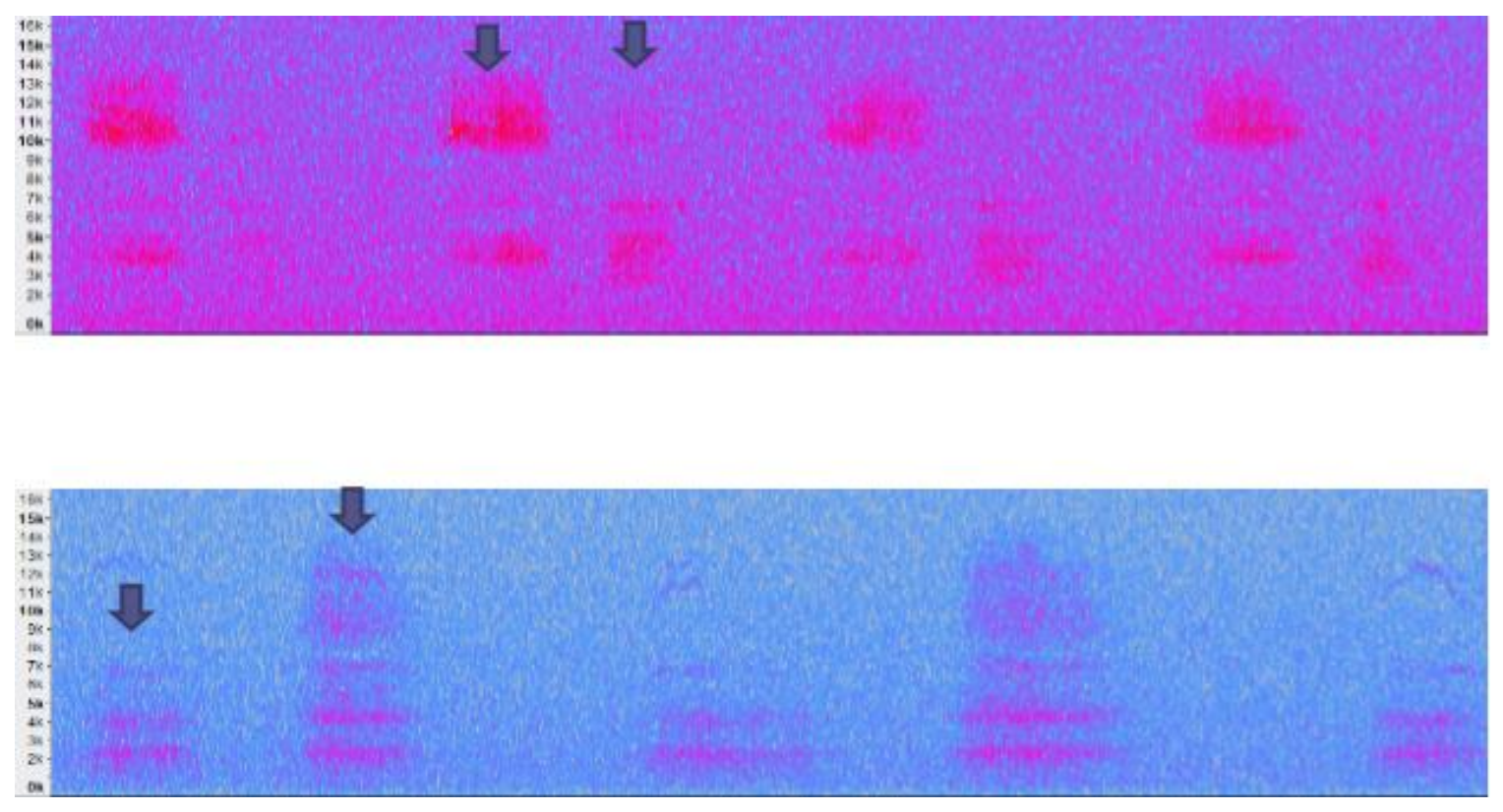

Figure 5 - Spectrograms from Adult and Child Breath Sounds

Between these two spectrograms, we can also see that there are many possible variables in the breath sounds. These include: 
- Overall amplitude of the breath sounds. An infant's breath will tend to be much quieter overall than an adult's, and the distance from the microphone will also affect this value. The sensitivity of the specific microphones used will also affect the amplitude of the breath sounds.

- Frequency range occupied by the breath sounds. A child's breath sounds will tend to be higher pitched, and much more of the relative amplitude will be in higher frequencies, some of which may not be reached by an adult breath sound at all. Furthermore, the range represented can vary slightly depending on the specific microphones used, as different microphones will be have different levels of sensitivity at different frequency ranges. A lower-quality microphone will have a less "flat" frequency response, meaning that it will tend to amplify certain frequency ranges more than others.

- Length of breath sounds. Some individuals may have shorter breaths than others, which means there will be a variable amount of time for the system to detect a breath on a given sound.

- Difference between inhalation and exhalation. We can see that the sounds of inhalation are generally quieter and lower frequency than exhalation sounds, but either are indicative of successful breathing. 
Input Signal Processing

Table 1 - Notation for Intermediate Values in LabVIEW

\begin{tabular}{|c|c|c|}
\hline Notation & Description & Derived from \\
\hline $\begin{array}{l}\text { Oraw, } \\
\text { Draw }\end{array}$ & $\begin{array}{l}\text { 2-D Omnidirectional and Directional } \\
\text { raw inputs, containing amplitude data } \\
\text { for all frequencies detected by the } \\
\text { microphone }\end{array}$ & $\begin{array}{l}\text { Raw input from Audio } \\
\text { Device }\end{array}$ \\
\hline $\begin{array}{l}\text { O }_{\text {filtered, }} \\
D_{\text {filtered }}\end{array}$ & $\begin{array}{l}\text { 2-D Filtered omnidirectional and } \\
\text { directional sound data, containing } \\
\text { amplitude data across all frequencies } \\
\text { within the bandpass filter }\end{array}$ & $\begin{array}{l}\text { Olraw or Dlraw passed } \\
\text { through } 2 \mathrm{kHz}-15 \mathrm{kH}- \\
\text { bandpass filter }\end{array}$ \\
\hline $\begin{array}{l}\text { OfRMS, } \\
\text { DfRMS }_{\text {fre }}\end{array}$ & $\begin{array}{l}\text { Decimal RMS value for } \\
\text { Omnidirectional or Directional filtered } \\
\text { input }\end{array}$ & $\begin{array}{l}\text { RMS value of } \mathrm{Ol}_{\text {filtered }} \text { or } \\
\text { Dlfiltered }\end{array}$ \\
\hline $\begin{array}{l}\text { ObRMS, } \\
\text { DbRMs }\end{array}$ & $\begin{array}{l}\text { Baseline RMS value for } \\
\text { Omnidirectional or Directional filtered } \\
\text { input }\end{array}$ & $\begin{array}{l}\text { Snapshot of Olfrims or } \\
\text { Dltrms without breath } \\
\text { sound }\end{array}$ \\
\hline $\begin{array}{l}\text { Onorm, } \\
\text { Dnorm }\end{array}$ & $\begin{array}{l}\text { RMS value of Omnidirectional or } \\
\text { Directional filtered input, normalized to } \\
\text { the baseline value }\end{array}$ & $\begin{array}{l}\text { OfRMs / ObRMS, DfRMs / } \\
\text { DbRMs }\end{array}$ \\
\hline RRMS & $\begin{array}{l}\text { Ratio of normalized Directional } \\
\text { compared to Omnidirectional }\end{array}$ & Dnorm / Onorm \\
\hline TRMS & $\begin{array}{l}\text { Threshold value for RRMs to qualify as } \\
\text { a breath }\end{array}$ & User input (see Testing) \\
\hline G current & $\begin{array}{l}\text { Current number of seconds between } \\
\text { breath sounds }\end{array}$ & $\begin{array}{l}\text { Elapsed time since the } \\
\text { last instant of RRMS }> \\
\text { TRMS }_{\text {RMS }}\end{array}$ \\
\hline Gapnea & $\begin{array}{l}\text { Seconds without breath before } \\
\text { "apnea" }\end{array}$ & User input \\
\hline
\end{tabular}

For the purposes of assigning a numeric value to any given moment of the audio signal, we use the instantaneous RMS. RMS refers to the root mean square of the signal at a given time. In an audio signal, this can be used to quantify the overall volume of an audio signal across a range of frequencies. It is 
important to use this function because breath sounds occupy a range of frequencies and may vary from breath to breath. If the device was to analyze the power of a single frequency, it would likely make the detections much less reliable. The RMS is calculated at location 1 in Figure 6.

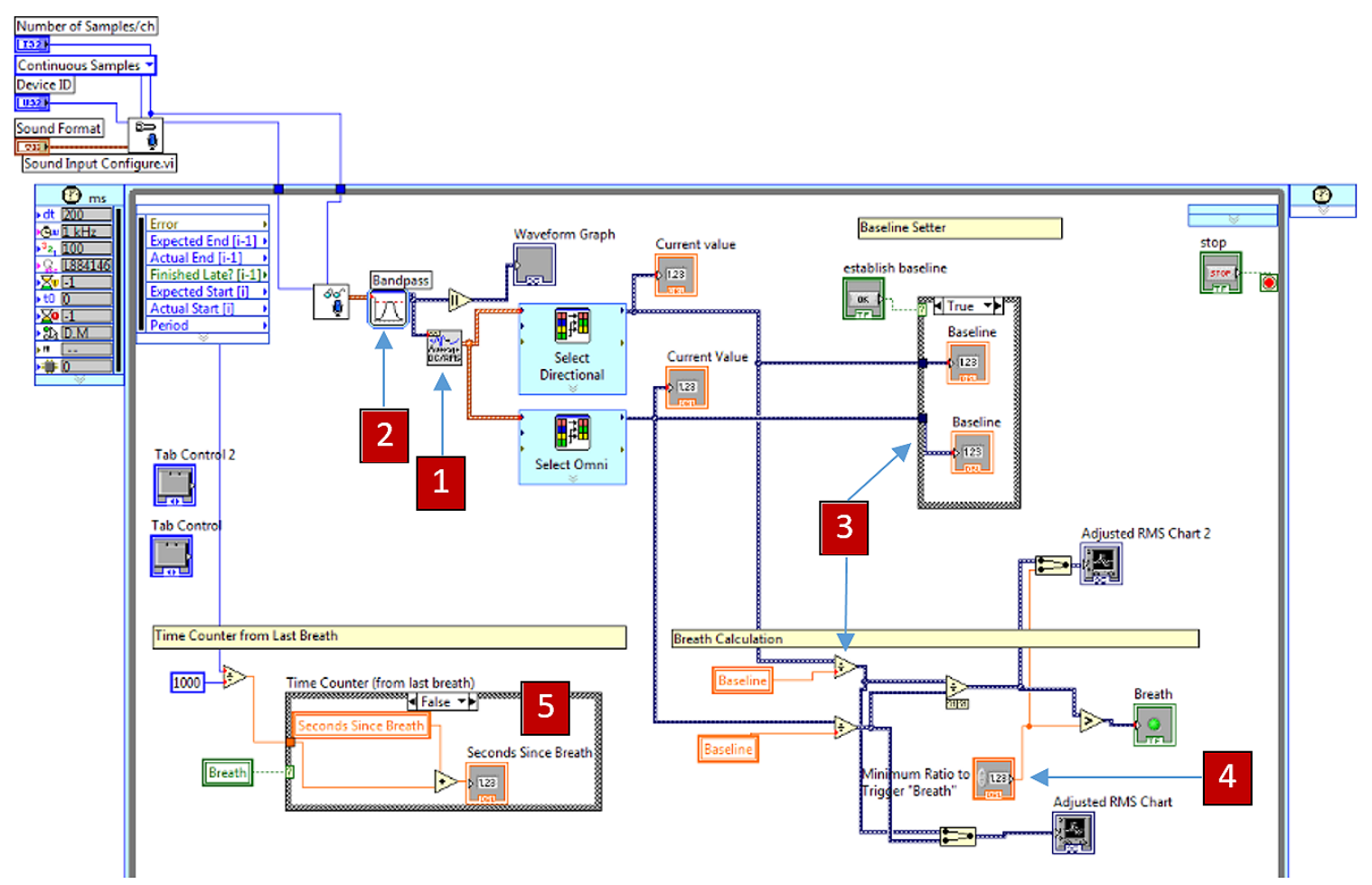

Figure 6 - LabVIEW Code

In addition, it would not be appropriate to analyze the entire range of audio that is detected by the microphone. As illustrated previously, the entire range would cover frequencies that could not contain sound produced by human 
breathing, which would in turn raise the RMS at each moment and reducing the significance of the RMS change initiated by the breath sound.

To eliminate that factor, the software uses a band pass filter, which removes frequencies from the audio signal that are either above or below the frequency ranges that were experimentally determined to possibly contain detectable breath sounds. From the spectrograms, a frequency range was selected that is wide enough to capture all possible breath sounds from a range of subjects and narrow enough to filter out constant noise or periodic noise unrelated to breath sounds. This particular band pass filter is between the ranges of $2 \mathrm{kHz}$ and $15 \mathrm{kHz}$. This is implemented at location 2 in Figure 6.

At this point we have a numerical value for the RMS at any instantaneous moment (OtRMs and DfRMs). In order for this value to be useful, we must determine the baseline non-breath sound to which it can be compared. Even after filtering and processing, there will still be non-breath noise in the targeted spectrum, and this software will use a ratio of this background RMS level to the currently detected RMS level to determine whether or not there is significantly increased volume in the targeted frequency range. Location 3 in Figure 6 includes the portion where the baseline is determined, as well as where the ratio of baseline to current RMS value is determined. The result is a numerical ratio for each channel (the omnidirectional Onorm and directional $D_{\text {norm}}$ ) that represents the strength of the potential breath sound. 
The next step is to take these two ratios - the relative filtered RMS value for the omnidirectional microphone and the relative filtered RMS value for the directional microphone - and compare them. If a loud noise in the room were to occur in the targeted frequency range, such as a loud car driving by, both microphones would detect it and we would see a spike in both ratios, but a quiet breath would only cause a spike in the directional microphone's ratio. Having two microphones allows us to essentially cancel out such a sound and only pay attention to the spikes that occur in our targeted zone, represented by the signal from the directional microphone. A single microphone solution would have no basis of comparison, and loud room noises would seem equally as significant as quiet breath noise. At location 4 in Figure 6, the instantaneous ratio between the value from each microphone ( $\mathrm{R}_{\mathrm{RMS}}$ ) is compared to the predetermined threshold value (TRMS), and results in a simple Boolean. A "yes" indicates that at the instantaneous moment, a significantly larger RMS spike in the directional microphone than the RMS spike in the omnidirectional microphone is occurring, and we determine this to be a breath (RRMS $>$ TRMS).

As discussed earlier, the clinical definition of apnea is based around the length of time between breaths. Therefore, the final step of signal processing is to take the Boolean of whether or not an instantaneous breath is occurring and use it to start a timer, which is represented at location 5 in Figure 6. Each time the Boolean breath value triggers a "yes", it resets the timer back to zero. The 
resultant output of this software is a numerical value indicating the number of seconds that have elapsed since the last breath sound.

\section{Handling of Processed Signal}

Figure 7 shows how the value of seconds since a breath signal is processed. The primary method that this data is used is to determine whether or not apnea is occurring in the subject. This is done by comparing the current number of seconds since breath (Gcurrent) against the programmed value consistent with the clinical definition of apnea (Gapnea), shown in location 1. This value can be adjusted to make the software mark an apnea event sooner or later, but because we are confident that every breath will instantaneously reset the timer, we leave that at the clinical value. Location 2 actuates the program-wide status of apnea / no apnea. 


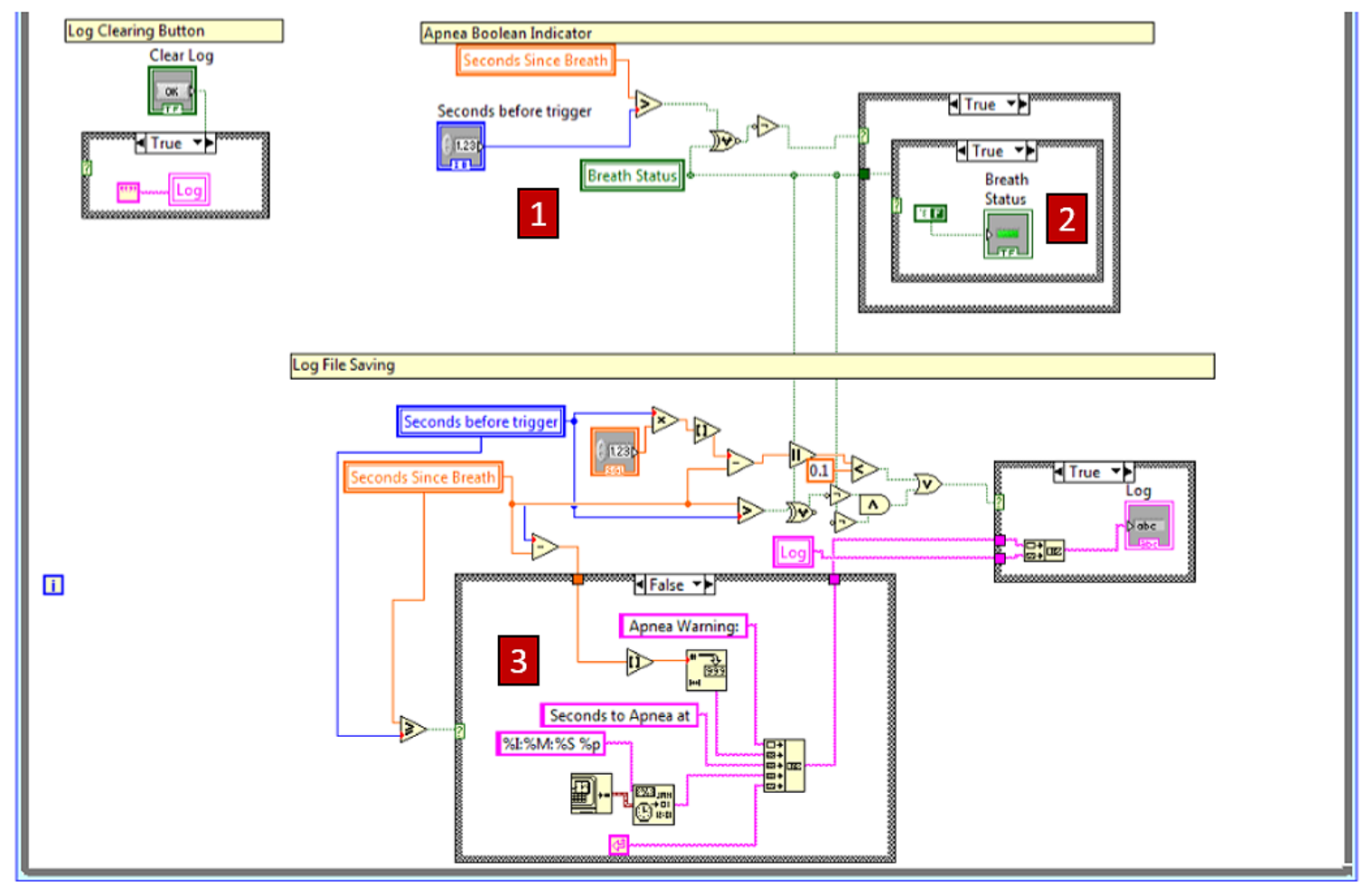

Figure 7 - LabVIEW Code, Continued

The section of the software at location 3 also includes a logging function. It will log events of apnea, along with the date and time that the apnea occurred. In addition, the software allows for an event of "apnea warning", which is an event where apnea almost occurred. This will allow the user to see if the subject is frequently getting very close to an apnea but not quite reaching it. The log data can be stored in a text file for long term review. 


\section{Front End - Graphs, Charts, and User Interface}

In Figure 8, the main feature is the large indicator bar at the bottom, which indicates the breath status. As long as the software detects a breath within the threshold time limit, it will remain green. Figure 9 shows an example of this same indicator bar when detecting an apnea event. The main interface can also display a number of graphs and raw data from the microphones. In this particular tab, we can see a chart of the adjusted ratios (R RMS) compared to the static threshold value (TRMs). Moments in time without a breath sound will dip below the threshold line, and moments with a breath sound will rise above it.

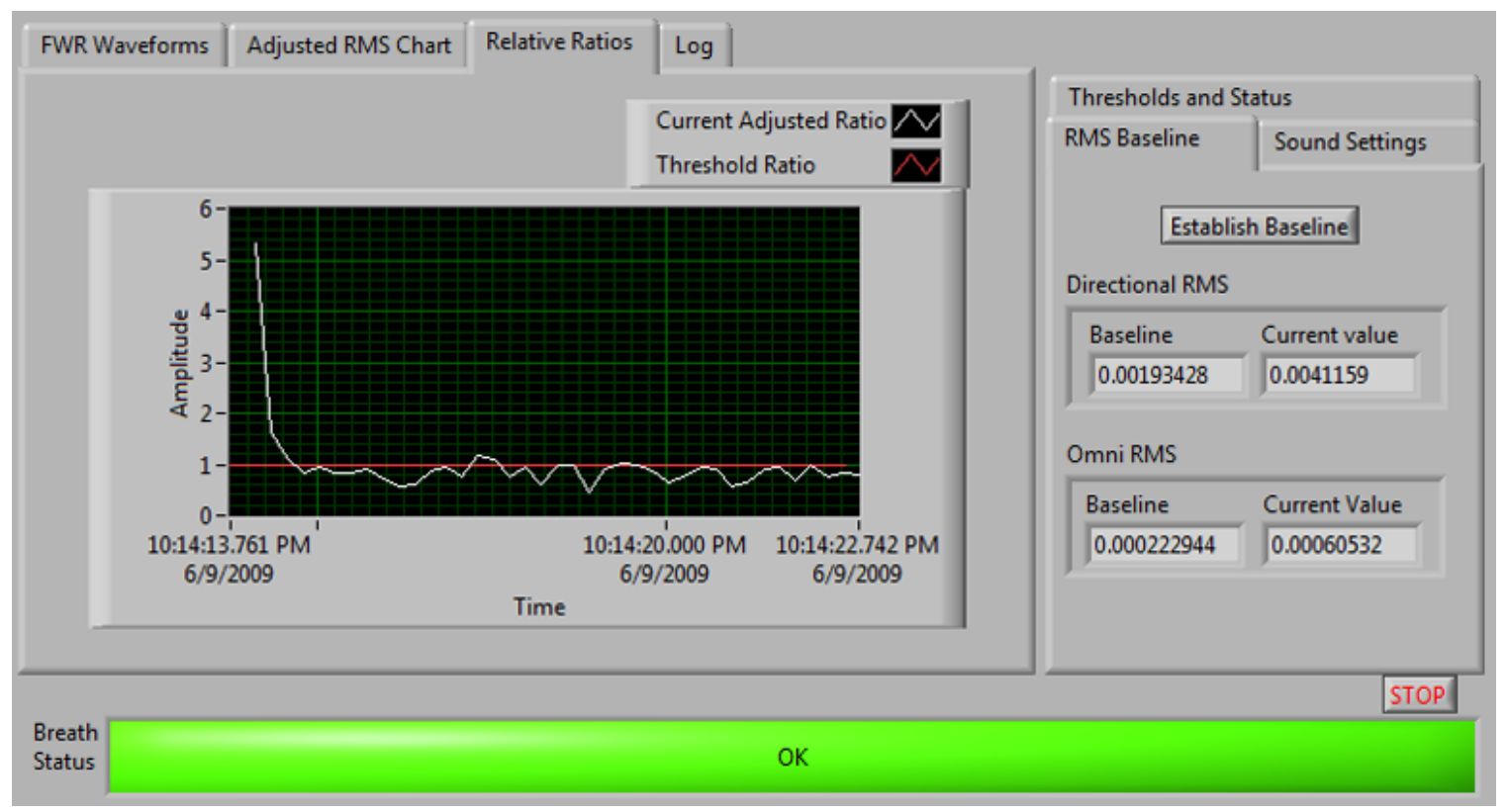

Figure 8 - LabVIEW User Interface 
In Figure 9, we see an example of the configurable settings. The log window is visible to the left, which shows recorded instances of apnea or near apnea, as well as the configuration for what near apnea events should be recorded. The controls at the right allow the user to configure the seconds without breath before an apnea event is logged as well as the triggering ratio (TrMS).

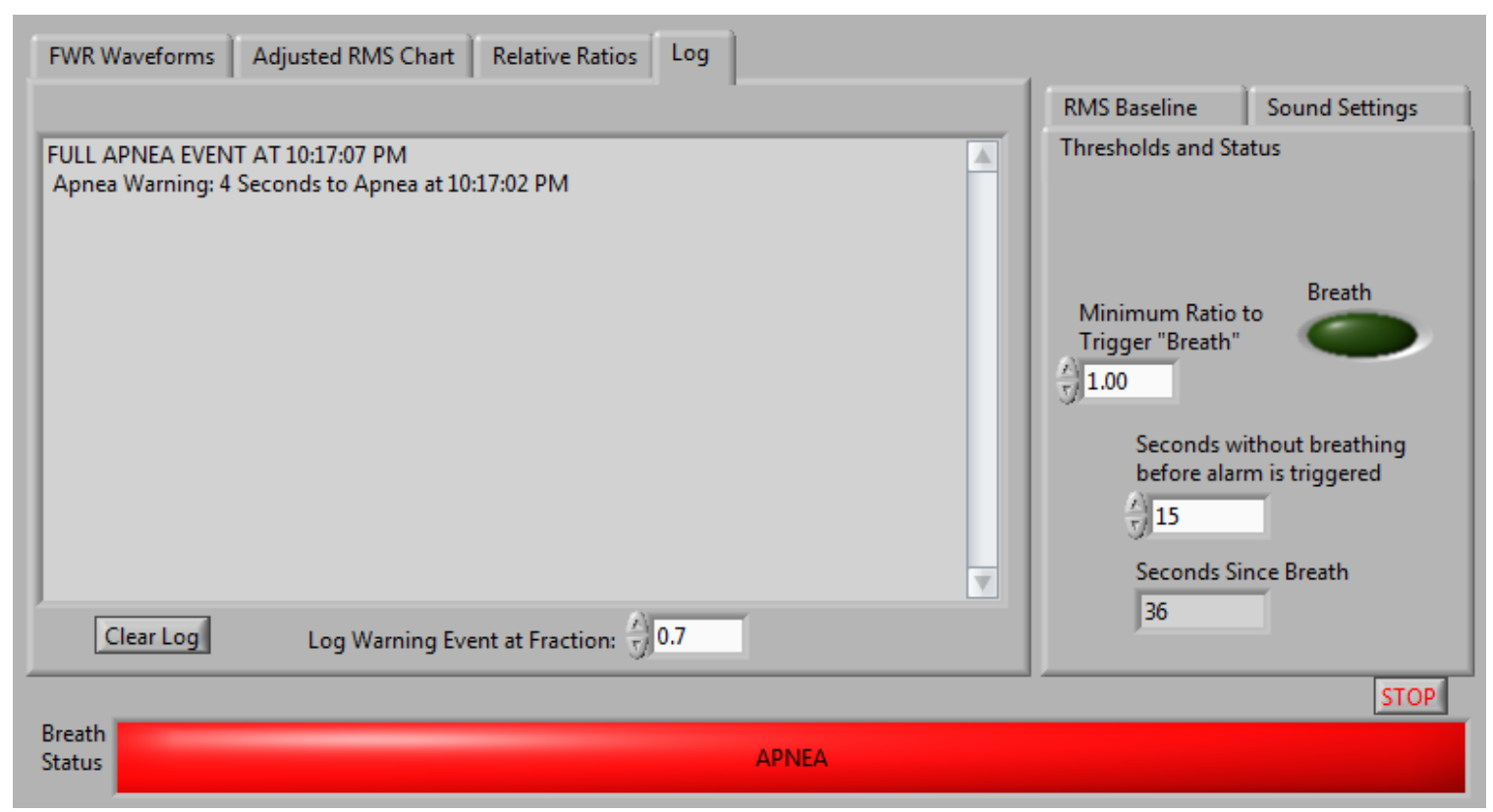

Figure 9 - LabVIEW Log Interface and Configurable Settings 


\section{TESTING}

Initial testing was performed to determine if the detection system was capable of reacting appropriately to common errors. Table 2 records the results of testing in which a scenario was forced that should trigger a reading and time delay to get the reading. For all tests, the system was set up to point at a healthy and breathing adult before the test scenario was introduced. Test type "breath held" indicates that the subject intentionally hold his breath until the test system reacted. "D. Microphone Unplugged" indicates that the directional microphone was disconnected from the system while monitoring. "Audio Device Unplugged" indicates that the USB cable connecting the audio device to the laptop was disconnected during testing. The "Time to State" column records the additional delay from the programmed apnea length $G_{\text {apnea }}$ before the system recorded an error or apnea.

Table 2 - Initial Error Testing Results

\begin{tabular}{|l|l|l|l|}
\hline Test & Type & State & Time to State \\
\hline $\mathbf{1}$ & Breath held & Apnea Detected & $+1 \mathrm{~s}$ \\
\hline $\mathbf{2}$ & Breath held & Apnea Detected & $+1 \mathrm{~s}$ \\
\hline $\mathbf{3}$ & Breath held & Apnea Detected & $+1 \mathrm{~s}$ \\
\hline $\mathbf{4}$ & Breath held & Detected & $+1 \mathrm{~s}$ \\
\hline $\mathbf{5}$ & D. Microphone Unplugged & Apnea Detected & $+1 \mathrm{~S}$ \\
\hline $\mathbf{6}$ & D. Microphone Unplugged & Apnea Detected & $+1 \mathrm{~S}$ \\
\hline $\mathbf{7}$ & Audio Device Unplugged & Apnea Detected & $+1 \mathrm{~s}$ \\
\hline $\mathbf{8}$ & Audio Device Unplugged & Apnea Detected & $+1 \mathrm{~s}$ \\
\hline
\end{tabular}


The results indicate that across all tests, the system indicated that an apnea was detected at Gapnea +1 second.

Table 3 records attempts to create errors in the system from false positives or false negatives while altering values $\mathrm{T}_{\mathrm{RMS}}$ (threshold ratio of normalized RMS values required to trigger a breath reading) and $G_{\text {apnea (number }}$ of seconds with no breath reading before apnea was declared). A healthy adult subject was used for all tests in this table. A false positive error means that the subject was breathing normally and the system recorded an apnea event (indicated by test type "+e"), and a false negative error means that the subject intentionally held his breath and the system did not record an apnea event (indicated by test type "-e"). "Attempts" is a count of the number of breaths taken

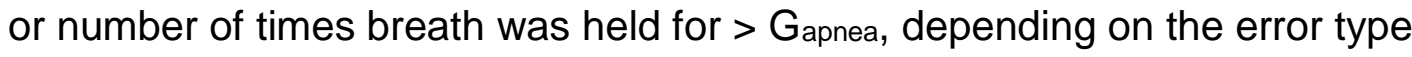
being tested.

Table 3 - Positive / Negative Error Testing Results

\begin{tabular}{|l|l|l|l|l|l|l|l|}
\hline Test & Type & Dist. & Duration & \multicolumn{1}{l|}{ Trms } & \multicolumn{1}{c|}{ Gapnea } & Attempts & Errors \\
\hline $\mathbf{1}$ & $+\mathrm{e}($ normal) & $1 \mathrm{ft}$ & $10 \mathrm{~min}$ & 1.05 & $20 \mathrm{sec}$ & 30 & 0 \\
\hline $\mathbf{2}$ & $-\mathrm{e}($ held) & $1 \mathrm{ft}$ & $5 \mathrm{~min}$ & 1.05 & $15 \mathrm{sec}$ & 15 & 2 \\
\hline $\mathbf{3}$ & $+\mathrm{e}($ normal) & $1 \mathrm{ft}$ & $10 \mathrm{~min}$ & 1.1 & $20 \mathrm{sec}$ & 30 & 0 \\
\hline $\mathbf{4}$ & $-\mathrm{e}$ (held) & $1 \mathrm{ft}$ & $5 \mathrm{~min}$ & 1.1 & $15 \mathrm{sec}$ & 15 & 0 \\
\hline $\mathbf{5}$ & $+\mathrm{e}($ normal) & $1 \mathrm{ft}$ & $10 \mathrm{~min}$ & 1.2 & $20 \mathrm{sec}$ & 30 & 7 \\
\hline $\mathbf{6}$ & $-\mathrm{e}$ (held) & $1 \mathrm{ft}$ & $5 \mathrm{~min}$ & 1.2 & $15 \mathrm{sec}$ & 15 & 0 \\
\hline
\end{tabular}


The results indicate that using an $R_{\text {RMS }}$ value of 1.1 recorded no positive or negative errors. An RRMs value of 1.05 resulted in negative errors, and an RRMS value of 1.2 resulted in positive errors.

Table 4 records results of a test scenario in which a loud heating fan was used to create a realistic and disruptive ambient noise at varying durations and distances (to vary the volume). The noise was created after normal monitoring had begun. During this time, the monitoring system was kept at a fixed distance of 3 feet from a healthy, breathing adult subject. The time without breathing sounds required to record an apnea event (Gapnea) was kept at a fixed value of 20 seconds.

Table 4 - Disruptive Ambient Noise Testing Results

\begin{tabular}{|l|l|l|l|}
\hline Test & Noise Distance & \multicolumn{1}{l}{ Noise Duration } & \multicolumn{1}{l|}{ Apnea Event } \\
\hline $\mathbf{1}$ & $1 \mathrm{ft}$ & 1 second & No \\
\hline $\mathbf{2}$ & $1 \mathrm{ft}$ & 5 seconds & No \\
\hline $\mathbf{3}$ & $1 \mathrm{ft}$ & 10 seconds & No \\
\hline $\mathbf{4}$ & $1 \mathrm{ft}$ & 25 seconds & Yes \\
\hline $\mathbf{5}$ & $1 \mathrm{ft}$ & 60 seconds & Yes \\
\hline $\mathbf{6}$ & $5 \mathrm{ft}$ & 1 second & No \\
\hline $\mathbf{7}$ & $5 \mathrm{ft}$ & 5 seconds & No \\
\hline $\mathbf{8}$ & $5 \mathrm{ft}$ & 10 seconds & No \\
\hline $\mathbf{9}$ & $5 \mathrm{ft}$ & 25 seconds & No \\
\hline $\mathbf{1 0}$ & $5 \mathrm{ft}$ & 60 seconds & No \\
\hline $\mathbf{1 1}$ & $20+\mathrm{ft}$ & 1 second & No \\
\hline $\mathbf{1 2}$ & $20+\mathrm{ft}$ & 5 seconds & No \\
\hline $\mathbf{1 3}$ & $20+\mathrm{ft}$ & 10 seconds & No \\
\hline $\mathbf{1 4}$ & $20+\mathrm{ft}$ & 25 seconds & No \\
\hline $\mathbf{1 5}$ & $20+\mathrm{ft}$ & 60 seconds & No \\
\hline
\end{tabular}


The results indicate that apnea events were only recorded when the disruptive noise was 1 foot from the monitoring system and the duration was 25 or 60 seconds.

Table 5 records the results of overnight testing of the complete system. The microphones were placed at a distance of 1-3 feet from a healthy adult male with no diagnosed history of apnea. The variance in that distance is due to potential movement of the subject during the night. "Software / Hardware Errors" indicates that an error caused the monitoring system to freeze, crash, or otherwise become inoperable.

Table 5 - Longevity Testing Results

\begin{tabular}{|l|l|l|l|l|}
\hline Test & Distance & \multicolumn{2}{c|}{ Duration } & \multicolumn{2}{c|}{ Apnea Events } & Software / Hardware Errors \\
\hline $\mathbf{1}$ & $1-3 \mathrm{ft}$ & 6 hours & 0 & 0 \\
\hline $\mathbf{2}$ & $1-3 \mathrm{ft}$ & 7 hours & 1 & 0 \\
\hline $\mathbf{3}$ & $1-3 \mathrm{ft}$ & 6 hours & 0 & 0 \\
\hline $\mathbf{4}$ & $1-3 \mathrm{ft}$ & 8 hours & 0 & 0 \\
\hline $\mathbf{5}$ & $1-3 \mathrm{ft}$ & 6 hours & 0 & 0 \\
\hline $\mathbf{6}$ & $1-3 \mathrm{ft}$ & 6 hours & 0 & 0 \\
\hline $\mathbf{7}$ & $1-3 \mathrm{ft}$ & 7 hours & 0 & 0 \\
\hline $\mathbf{8}$ & $1-3 \mathrm{ft}$ & 6 hours & 0 & 0 \\
\hline $\mathbf{9}$ & $1-3 \mathrm{ft}$ & 6 hours & 0 & 0 \\
\hline $\mathbf{1 0}$ & $1-3 \mathrm{ft}$ & 6 hours & 0 & 0 \\
\hline
\end{tabular}


The results indicate that the monitoring system did not experience any software or hardware errors that caused the system to become inoperable during the 10 nights of testing. On one 7-hour night of testing, an apnea event was recorded, but this reading was not reproduced in any of the subsequent nights of testing.

Table 6 records the results of the use of the complete monitoring system in the Neonatal Intensive Care Unit (NICU) at Cottage Hospital in Goleta, CA under the supervision of Dr. Steven Barkley. A single prematurely born infant was used across all three tests who did not currently have difficulty breathing or apnea. At a distance of 6 inches, no apneas or near apneas were recorded by the system. At a distance of 12 inches, 3 apneas were recorded and 8 near apneas were recorded. During the testing at 24 inches, the subject began to move around, rustling the sheets in the process, which the monitoring system recorded as no apneas or near apneas.

Table 6 - Premature Infant Testing Results

\begin{tabular}{|l|l|l|l|l|}
\hline Test & Duration & Distance & Apneas & Near Apneas \\
\hline $\mathbf{1}$ & $5 \mathrm{~min}$ & 6 in & 0 & 0 \\
\hline $\mathbf{2}$ & $5 \mathrm{~min}$ & 12 in & 3 & 8 \\
\hline $\mathbf{3}$ & $5 \mathrm{~min}$ & 24 in & $0^{*}$ & $0^{\star}$ \\
\hline
\end{tabular}




\section{DISCUSSION}

\section{Discussion of Table 2 - Initial Error Testing Results}

The purpose of this test was to both establish effectiveness of the expected results from a sudden lack of breathing as well as record results of unexpected disconnection. This could only be performed on an adult capable of holding their breath for longer than $G_{a p n e a}$ at 20 seconds, and the results indicate that the device is fully functional in this scenario. Adults represent the best-case scenario with louder breathing noises than children or infants, and this test establishes this level of minimum effectiveness.

When disconnecting microphones or the Audio Device, the monitoring system does not immediately show an error, but it does fail to detect breath sounds, resulting in the detection of an apnea event once $G_{\text {apnea }}$ is exceeded. Clearly, this is not a correct reading, but it can be safely assumed that if this scenario were to set off the apnea alarm, a caretaker would still want to know that the system had been connected so that they could resume continuous monitoring, and the alarm therefore still serves a purpose. 


\section{Discussion of Table 3 - Positive / Negative Error Testing Results}

The purpose of this test is to establish realistic relative threshold values (T $\mathrm{RMS}$ ) such that the rate of positive and negative errors is minimized. A TRMS value that is too high requires an unrealistically loud noise from the directional microphone to trigger a breath in the monitoring system, which is why we can see it results in a false positive. On the other hand, a value of Trms that is too low will result in the monitoring system constantly detecting a breath from inherent fluctuations in the sound recording and not necessarily tied to actual breath sounds, which is why we can see it results in a false negative. Of the two possible scenarios, we would prefer to err on the side of $\mathrm{T}_{\mathrm{RMS}}$ being too high, as it is more unacceptable to miss a real apnea event than it is to trigger an alarm while the subject is breathing.

These results indicate that a $T_{\text {RMS }}$ value allowing functional operation does exist with the low-cost audio equipment in this monitoring system. These numbers are heavily influenced by the clarity of signal coming from the microphones and USB audio device. The more effective the directional microphone is at attenuating outside noise, the higher R RMS (instantaneous RMS ratio between microphones) will be from breath sounds, and the higher $\mathrm{T}_{\text {RMS }}$ can be, resulting in a more accurate system. In addition, higher quality microphones and audio devices will also include less inherent signal noise, which would have the same effect. 


\section{Discussion of Table 4 - Disruptive Ambient Noise Testing Results}

The purpose of this test is to investigate the effectiveness of the noise cancelling system provided by the use of both a directional and omnidirectional microphone. A realistic source of noise, a loud heating fan, was turned on suddenly during monitoring, which might represent a real world scenario of a room's heating or air conditioning engaging during the night, or a loud car driving past.

The results show that the dual microphone system is effective at continuing to monitor breaths without a false positive in all scenarios except for a particularly lengthy (> Gapnea) and loud noise. In order for that to occur in a real world scenario, the monitoring system would have to be placed almost on top of a potentially loud noise source, which could easily be avoided with proper instruction to caretakers.

\section{Discussion of Table 5 - Longevity Testing Results}

The longevity testing results cover 10 nights of continuous monitoring. On the second night of testing, the system recorded an apnea event, but this did not occur in any of the other nights of testing. This could indicate an error in the detection system, but it is possible that this was actually at true apnea event. 
More importantly the test indicates that the monitoring system is stable enough to run continuously through the night without encountering any sort of hardware or software errors that would disable the test (memory leak, computer going to sleep, etc).

Combined with the results from the previous test cases, we can have confidence that this system is capable of effective and long-term monitoring of an adult subject.

\section{Discussion of Table 6 - Premature Infant Testing Results}

The previous testing results established the effectiveness of the monitoring system on a best case scenario - a healthy adult. It was very important that data also be gathered from infants in order to assess the effectiveness of the monitoring system on a much quieter subject, especially considering the fact that infants are the most likely subjects in a real-world scenario. The infants at the NICU in Goleta represent the worst case scenario, as they are even quieter and tougher to detect than a larger full-term infant. On top of that, the NICU environment was filled with ambient noises of machinery and HVAC, lessening the impact of the breath sounds even more.

The results, although very limited in scope, are very promising. Going into the test, there was little confidence that anything would be detected. The results 
indicated that at the 6 inch range, the system was effective in detecting consistent breaths. One qualitative result from the experiment that was not recorded in the table is that during the test, it was visually verified that the monitoring system's indications of a breath matched up with actual breaths by the infant.

The data from 12 inches indicates that the system is not without limitations, and for a subject as quiet as a premature infant, the clarity and sound isolation of this monitoring system were not enough to confidently detect breaths. However, given the results at 6 inches, it is very possible that with further software tweaks or more precise equipment, the monitoring system could be effective at the longer and more realistic ranges of 12 inches and beyond. 


\section{CHALLENGES AND FUTURE STEPS}

\section{Subjects}

An ongoing challenge with the project was getting good subjects. Tests were performed on adult members of the project team, aside from the testing performed in the NICU. Adult subjects have the advantage of being able to hold their breath, but don't represent the most likely use case of a commercial product of this nature. The ideal scenario would be testing on newborn infants that were known to have issues with sleep apnea, but no such subjects were available, and it would require a redundant monitoring system in order to verify the results of the system in this project.

Future projects should focus heavily on infant testing as early as possible. For this project, we were lucky to get access to an infant in the NICU at all and the data was extremely valuable.

\section{Equipment}

Equipment for this project was selected off the shelf and underneath budget constraints. As discussed earlier, a higher quality set of microphones would allow for a more precise measurement and more effective attenuation of off-axis sound by the directional microphone, which would in turn result in more reliable measurements. For instance, Audio Technica produces a microphone of 
similar type to the directional microphone used in this project (model number BP4071L) which would provide approximately five times the voltage output from a given sound. If a product like this were to go to market, the savings of bulk purchase would allow much higher quality microphones to be used.

In addition, it should be noted that the actual computations involved with this LabVIEW program are not complicated and could be executed on much more rudimentary hardware, such as an Arduino microcontroller kit. Migrating to a kit like this would not only drive down costs of the unit by more than half, but would greatly decrease the weight and footprint, allowing for even more flexible placement.

Some of the potential detection systems were also excluded partially on the basis of cost. If the project was to have a higher budget, more detection systems would be viable for the project. Also, some of the technologies (FLIR, in particular) are getting much less expensive as time goes by, and may be financially viable for home monitoring before long.

\section{Software}

The LabVIEW software used does require configuration before monitoring can safely begin, most notably the establishment of the baseline reading. With more time, it would be possible to create software that established this baseline 
automatically, which would reduce the amount of user input as well as the possibilities for user error.

The LabVIEW software would also be useful for testing known breath scenarios if it had the ability to analyze a pre-recorded sound session. With this functionality, the program could be tweaked and repeated on the same apnea event until it is detected properly.

One potential feature of the software that was discussed but was not implemented during this project was the ability to record audible samples from apnea events. This would require the software to be continuously recording a floating window of sound data which would then be saved with a timestamp after an apnea event is triggered. Alternatively, a simple spectrogram image of that time stamp could be used. This feature would be useful in determining whether or not a true apnea occurred, and beyond that, determining if other noises were occurring simultaneously that might indicate struggling from obstructive apnea.

\section{Skin Contact}

The most restrictive design requirement in this project was the requirement to eliminate skin contact with the subject. If this requirement were to be removed, it may be much simpler to acquire accurate sound data from the subject. For instance, microphones could be installed in the clothing worn by an 
infant, or directly attached to the skin, which would eliminate any ambiguity in the source of the sound and drastically reduce false positives. 


\section{CONCLUSION}

This project has demonstrated that off the shelf and affordable components can be used to create a new monitoring system that accurately detects lapses in breathing in adults and infants. Future projects will be capable of building upon this work and increase the accuracy in subjects that are difficult to detect. Furthermore, it is very possible for a commercialized version of this monitor to be even more cost effective and accurate. 


\section{WORKS CITED}

1. Abu-Shaweesh, J.M, \& Martin, R.J. (2008). Neonatal Apnea: What's New?. Pediatric Pulmonology, 2008 (43), 937 - 944.

2. Guyton, A.C., \& Hall, J.E. (2006). Textbook of Medical Physiology (1 $11^{\text {th }}$ Edition.). Philadelphia, Pennsylvania: Elsevier Saunders.

3. Hafner, N., \& Mostafanezhad, I., \& Lubecke, V. M., \& Boric-Lubecke, O., \& Host-Madsen, A. (2007, August 23-26). Non-Contact Cardiopulmonary Sensing with a Baby Monitor. Paper presented at the 29th Annual International Conference of the IEEE EMBS: Engineering in Medicine and Biology Society, Lyon France. doi:10.1109/IEMBS.2007.4352785

4. Hall, K.L., \& Zalman, B. (2005). Evaluation and Management of Apparent Life-Threatening Events in Children. American Family Physician, 71 (12).

5. Leiter, J.C., \& Böhm, I. (2007). Mechanisms of pathogenesis. Respiratory Physiology \& Neurobiology , 2007 (159), 127-138.

6. Malakuti, K., \& Albu, A.B. (2010, August 23-26). Towards an Intelligent Bed Sensor: Non - Intrusive Monitoring of Sleep Irregularities with Computer Vision Techniques. Presented at $20^{\text {th }}$ International Conference on Pattern Recognition, Istanbul. doi: 10.1109/ICPR.2010.974

7. Mishra, S., \& Agarwal, R., \& Jeevasankar, M., \& Aggarwal, R., \& Deorari, A.K., \& Paul, V.K. (2008). Apnea in the Newborn. Indian Journal of Pediatrics, 2008 (75), 57-61.

8. Moon, R.Y., \& Horne, R.S.C., \& Hauck, F.R. (2007). Sudden Infant Death Syndrome. Lancet, 2007 (370), $1578-87$.

9. Nishada, Y., \& Mori, T., \& Sato, T., \& Hirai, S. (2009, October 12-15). The surrounding Sensor Approach - Application to Sleep Apnea Syndrome Diagnosis based on Image Processing. Paper presented at IEEE International Conference on Systems, Man, and Cybernetics, Tokyo. doi: 10.1109/ICSMC. 1999.816582 
10. Thach, B.T. (2005). The role of respiratory control disorders in SIDS. Respiratory Physiology \& Neurobiology, 2005 (149), 343-353.

11. Wildhaber, J.H., \& Moeller A. (2007). Sleep and respiration in children: time to wake up!. Swiss Med Wkly, 2007 (137), 689-694.

12. West, L. J. (2010). A standoff Approach to Monitoring Infant Apnea (Unpublished Masters Thesis). California Polytechnic State University, San Luis Obispo, CA. 